\title{
Virtual Element Methods for the Spatial Discretisation of the Multigroup Neutron Diffusion Equation on Polygonal Meshes with applications to Nuclear Reactor Physics
}

\author{
J.A. Ferguson, J. Kópházi, M.D. Eaton \\ Nuclear Engineering Group, Department of Mechanical Engineering, City and Guild Building, Imperial \\ College London, Exhibition Road, South Kensington Campus, London, SW7 2BX, United Kingdom
}

\begin{abstract}
The Continuous Galerkin Virtual Element Method (CG-VEM) is a recent innovation in spatial discretisation methods that can solve partial differential equations (PDEs) using polygonal (2D) and polyhedral (3D) meshes. This paper presents the first application of VEM to the field of nuclear reactor physics, specifically to the steady-state, multigroup, neutron diffusion equation (NDE). In this paper the theoretical convergence rates of the CG-VEM are verified using the Method of Manufactured Solutions (MMS) for a reaction-diffusion problem in the presence of both highly distorted and non-convex elements and also in the presence of discontinuous material data. Finally, numerical results for the 2D IAEA and the 2D C5G7 industrial nuclear reactor physics benchmarks are presented using both block-Cartesian and general polygonal meshes.
\end{abstract}

\section{Introduction}

The modern design and analysis of nuclear reactor cores, within contemporary nuclear power plants (NPPs), requires the solution of a complex transport equation which models the migration and interaction of neutrons with the materials within the nuclear reactor core [1]. This neutron transport equation (NTE) is a partial-integro-differential equation (PIDE) which is a function of a seven-dimensional phase of solution variables which are: position $(x, y, z)$, energy $(E)$, angle $(\theta, \chi)$ and time $(t)[2]$. A mathematical approximation to the NTE is often used in whole core nuclear reactor physics simulations and is called the neutron diffusion equation (NDE). The NDE is derived from the NTE by assuming that the angular variation of neutrons is linearly-anisotropic. Using this approximation to the angular variation of neutrons means that the NDE is only a function of position $(x, y, z)$, Energy $(E)$ and time $(t)$ [3]. This means that the NDE is much easier to solve than the NTE for whole core nuclear reactor physics simulations. The NDE is a reasonable approximation to the NTE for neutron migration in weakly absorbing media several mean free paths away from any isolated sources, boundaries of the domain or at the interfaces between materials with significantly different material properties [3]. The focus of this paper will be the solution of the NDE for various nuclear reactor physics verification test cases using the virtual element method (VEM) which is a relatively recent innovation in spatial discretisation techniques [4].

Email address: jaf13@ic.ac.uk (J.A. Ferguson, J. Kópházi, M.D. Eaton) 
A typical industrial nuclear reactor design process involves multiple stages, beginning with the solution of the multi-group NTE on a single nuclear reactor fuel assembly for a large number of energy groups, this is commonly referred to as the nuclear reactor lattice calculation [5]. The solution from the lattice calculation is then homogenised over space and condensed over energy to reduce the number of energy groups to between 2-4 energy groups [6, 7]. The space-homogenised and energy-condensed macroscopic neutron cross-sections are then used to perform whole-core nuclear reactor physics simulations; which typically involves solving the multi-group NDE. Spatial discretisation of the space-homogenised multi-group NDE has typically been accomplished using nodal methods $[5,8]$. The nodal method is a locally conservative, coarse Cartesian mesh, discretisation method that calculates integral quantities over volumes and surfaces such as reaction rates in a region and net currents in or out of a "control volume" or "node". Usually the "control volume" or "node" is an entire nuclear fuel assembly with homogenised material properties. The homogenisation of nuclear fuel assembly material properties necessitates a loss of spatial information of the solution field. If a high-fidelity, geometry conforming, spatial discretisation method is required to solve the multi-group NTE the most widespread approach, adopted by industry, is the method of characteristics (MoC) [9]. The main alternative to the $\mathrm{MoC}$ is the finite element (FE) method which saw its first uses in nuclear reactor physics in the early 1970s [10, 11].

In recent years the FE method has been extended to include adaptive mesh refinement (AMR) algorithms with the adaptivity driven by dual weighted residual (DWR) error estimators [12, 13]. The benefits of the FE method over other spatial discretisation methods such as finite differences (FD) and nodal methods is the ability to discretise differential operators using unstructured meshes. Therefore, the mesh can more closely approximate the complex geometry of the problem when compared to the purely Cartesian meshes employed in FD and nodal methods. However, the FE method is limited to meshes comprised of triangles or quadrilaterals in $2 \mathrm{D}$ and tetrahedra, prisms, pyramids or hexahedra in 3D. In addition to this limitation the convergence properties degrade in the presence of highly distorted or stretched elements [14]. Nuclear reactor design often generates very complex geometries in which geometric details at small length scales relative to the size of the full nuclear reactor core have to be represented by the mesh. Representing these details with a FE mesh can result in highly distorted elements that can cause degradation of the CG-FEM solution [15]. Determining when this happens and performing operations to improve the quality of the FE mesh can be challenging; albeit AMR approaches can perform some of these operations [16].

It has been estimated that geometry decomposition, meshing and mesh manipulation can take up to $77 \%$ of the total analysis time [17]. Isogeometric analysis (IGA) is a spatial discretisation method that attempts to streamline the computer aided design (CAD) to computer aided engineering (CAE) analysis process by removing the mesh generation step altogether. It uses the same basis functions that are used by CAD software to represent the geometry of the physical domain [17]. Therefore, in theory, an engineer could perform CAE analysis of the physical problem directly on the geometric description of their engineering design without any processing by another software package to perform ancillary mesh generation. Research has been conducted on applying IGA spatial discretisation methods to nuclear reactor physics problems $[18,19,20$, $21,22,23,24]$. Other attempts to mitigate or ameliorate the challenge of mesh generation have involved relaxing the strict requirements on mesh topology by constructing polygonal and polyhedral spatial discretisations. These methods include the polygonal finite element method (PFEM) [25, 26, 27, 28], the piecewise linear finite element discretisation (PLFE) $[29,30]$ and the mimetic finite difference method (MFD) [31, 32, 33, 34, 35]. Each of these methods, while solving the problem of restrictive mesh topology, have their own limitations. Within both the PFEM and PLFE methods higher order schemes are impossible while in high-order MFD 
the method is complex compared to Galerkin schemes. The virtual element method (VEM) represents a viable alternative to all of these methods.

The VEM is a relatively recent innovation in spatial discretisation methods which has been used to solve a wide range of partial differential equations (PDEs) [4]. Its mathematical formulation is largely the same as the FEM; however it utilises many of the concepts developed in the MFD literature. In particular the VEM has inherited the notion of a discrete field from MFD whereby degrees of freedom (DoFs) are distributed to topological entities such as vertices, edges, faces and cells which are then used to define discrete bilinear forms. The MFD formulation that most closely resembles the VEM presented in this paper may be found in [31], which was the extension of MFD to arbitrary order for elliptic systems. However, the VEM is sufficiently different to MFD to merit the creation of a new name with a number of recent publications within the literature. The central idea in VEM is to take what are effectively MFD DoFs but to map shape functions to them explicitly [4]. The form of these shape functions is not known on the interior of the element except that they are composed of both polynomial and non-polynomial parts [4]. The method avoids performing integrations on the shape functions directly by using projections onto the space of polynomials that may be computed using the degrees of freedom alone [4]. Because the shape functions need not be known explicitly on the interior of an element the VEM has far greater flexibility in terms of element geometry compared to the finite element method.

Approximation spaces of arbitrary order and arbitrary regularity [36] may be constructed on very general element geometries and numerous numerical experiments demonstrate the robustness of the VEM to element degeneracy. Thus far various aspects of VEM theory have been developed including the choice of internal DoFs [37], the development of projection operators [38], VEM with arbitrary regularity [36], higher order VEM on polyhedral meshes [39], nonconforming VEM [40, 41, 42, 43] and SUPG stabilised VEM [43, 44]. Additionally H(div) and $\mathrm{H}$ (curl) conforming VEM were presented in [45]. More recently some effort has been expended in developing orthogonal polynomial bases and alternative stabilisation terms for higher order $\operatorname{VEM}[46,47,48]$. The main reason for this research is the mitigation of ill-conditioning for higher order VEM schemes. The VEM has been applied to numerous problems in science and engineering including general second order elliptic problems in both primal and mixed form [49, 50], hyperbolic problems [51], parabolic problems [52], polyharmonic problems [53] and biharmonic problems [54]. Additionally some applied problems have been presented such as linear elasticity and plate bending problems $[55,56]$, friction and contact problems $[57,58]$. This list is far from exhaustive but provides a sufficient overview of the current range of applicability of the VEM in science and engineering.

The next section will discuss the continuous Galerkin formulation of the VEM (CG-VEM). Section 3 will discuss the CG-VEM discretisation procedure for the multi-group NDE, including a few details on how the final block matrix system of equations is assembled. Section 4 presents two numerical verification tests: the 2D IAEA benchmark and the 2D OECD-NEA C5G7 benchmark. These problems were solved with various types of meshes in order to demonstrate the versatility of the VEM with respect to mesh topology. The centroidal Voronoi tessellation algorithm [59, 60], is a computationally efficient and numerically robust mesh generation algorithm which is used to generate the polygonal meshes presented in this paper. These types of mesh generation algorithms can tessellate very complex 2D and 3D geometrical domains. Therefore, this approach can reduce and ameliorate the challenge of mesh generation which was described earlier in this paper, some recent advances may be found in [61, 62, 63, 64, 65]. A method of manufactured solutions (MMS) is presented in the appendix to verify the order of convergence of the CG-VEM in the presence of both element distortion and concavity. Additionally, an MMS involving a reaction-diffusion equation with piecewise-constant coefficients 
and piecewise-smooth forcing function was performed. Section 5 will provide a discussion of results and some concluding thoughts.

Note, that we presented an early version of the research presented in this paper at the international conference on nuclear engineering (ICONE) in 2018 which was hosted in London, United Kingdom (UK). The conference proceedings may be found in [66].

\section{The Virtual Element Method (VEM)}

\subsection{Preliminaries}

Vectors in $\mathbb{R}^{n}$ are denoted by lower case bold e.g $\mathbf{v}$ and matrices in $\mathbb{R}^{m \times n}$ are denoted in upper case bold e.g A. The element at index $i$ of a vector or $(i, j)$ of a matrix are denoted by $(\mathbf{v})_{i}$ and $(\mathbf{A})_{i, j}$ respectively. Let $D \subset \mathbb{R}^{d}$ and the boundary, centroid, measure and diameter of $D$ be denoted by $\partial D, \mathbf{x}_{D}, A_{D}$ and $h_{D}$ respectively. The $H^{s}(D)$ norm and semi-norm on $D$ are denoted by $\|\cdot\|_{s, D}$ and $|\cdot|_{s, D}$ and the $L^{2}(D)$ inner product is denoted as $(\cdot, \cdot)_{0, D}$. The notation $[a, b]$ is used to denote either closed set of real numbers between $a$ and $b$ or the set of consecutive integers $\{a, a+1, \ldots, b\}$, the context should make clear which meaning is taken. Let the scaled monomials, $m_{\alpha}(\mathbf{x})$, in $\mathbb{R}^{d}$ be defined as:

$$
m_{\alpha}(\mathbf{x})=\prod_{i=1}^{d}\left(\frac{\left(\mathbf{x}-\mathbf{x}_{D}\right)_{i}}{h_{D}}\right)^{\alpha_{i}}
$$

where there is a bijective mapping between a linear index $\alpha$ and multi-index $\boldsymbol{\alpha}=\left(\alpha_{1}, \ldots, \alpha_{d}\right)$. Let $|\boldsymbol{\alpha}|=\alpha_{1}+\ldots+\alpha_{d}$, then set of scaled monomials on $D$ of order $k$ is defined as:

$$
\mathcal{M}_{k}(D)=\left\{m_{\alpha}(\mathbf{x}), \quad 0 \leq|\boldsymbol{\alpha}| \leq k\right\}
$$

The set of scaled monomials with order strictly equal to $k$ shall be denoted by $\tilde{\mathcal{M}}_{k}(D)$. The set of polynomials of order less than or equal to $k$ is denoted by $\mathcal{P}_{k}(D)$ and of order strictly $k$ by $\tilde{\mathcal{P}}_{k}(D)$. In this paper $\mathcal{M}_{k}(D)$ is taken as the basis of $\mathcal{P}_{k}(D)$ therefore the dimension of $\mathcal{P}_{k}(D)$, denoted by $n_{k}$, is given by:

$$
n_{k}=\frac{(k+d) !}{k ! d !}
$$

The scaled monomial basis of vector-valued polynomials $\left[\mathcal{P}_{k}(D)\right]^{d}$ is denoted by $\mathcal{M}_{k}^{d}(D)$ and in two-dimensions (2D) may be expressed as:

$$
\mathcal{M}_{k}^{2}(D)=\left\{\mathcal{M}_{k}(D) \times\{\mathbf{0}\}\right\} \cup\left\{\{\mathbf{0}\} \times \mathcal{M}_{k}(D)\right\}
$$

Members of $\mathcal{M}_{k}^{d}(E)$ are denoted $\mathbf{m}_{\alpha}$ where $\alpha \in\left[1, d \times n_{k}\right]$.

\subsection{The Local Space}

The description of the CG-VEM provided by this section will be restricted to the $2 \mathrm{D}$ case and is based entirely on work first published in [4, 38, 49, 67]. Let $V \subset \mathbb{R}^{2}$ be some arbitrary domain with a piece wise continuous boundary $\partial V$ and let $\mathcal{T}_{h}$ be a decomposition of $V$ into a set of simple, non-overlapping polygons. Following [49], for each polygon $E$ in the mesh there must 
exist a $\gamma>0$ such that the polygon is star-shaped with respect to a ball of radius $\gamma h_{E}$ (for a more detailed description of what this means see [68], section 4.2.2). The length of each edge $e \in \partial E$ must satisfy $|e| \geq \gamma h_{E}$. An example of such a mesh is shown in figure 1 .

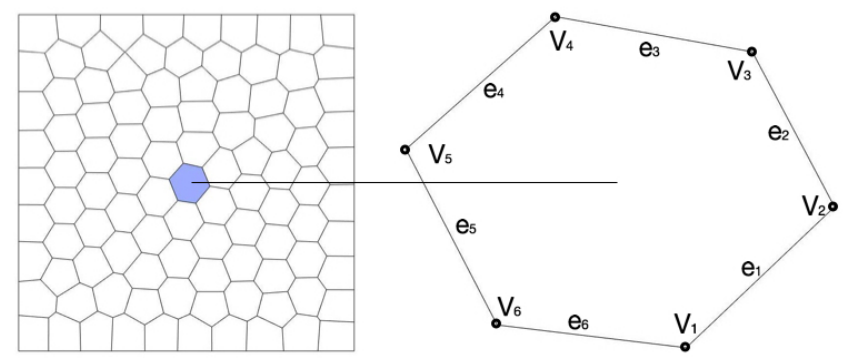

Figure 1: An example decomposition of a unit square into polygonal elements.

Considering a polygon $E \in \mathcal{T}_{h}$ the initial local virtual element space $\tilde{\mathcal{V}}_{k}(E)$ is defined as [49]:

$$
\tilde{\mathcal{V}}_{k}(E)=\left\{v_{h} \in H^{1}(E):\left.v_{h}\right|_{e} \in \mathcal{P}_{k}(e) \forall e \in \partial E, \Delta v_{h} \in \mathcal{P}_{k}(E)\right\}
$$

Supposing $E$ has $N_{v}$ vertices then for any $v_{h} \in \tilde{\mathcal{V}}_{k}(E)$ the following set of linear functionals $\operatorname{dof}_{i}\left(v_{h}\right): \tilde{\mathcal{V}}_{k}(E) \rightarrow \mathbb{R}$ are defined on the local CG-VEM function space [49]:

1. The value of $v_{h}$ at each vertex of $E$.

2. The value of $v_{h}$ at the interior $k-1$ Gauss-Lobatto points on a on each edge of $E$.

3. The internal moments with scaled monomials of order $k-2$ :

$$
\frac{1}{|E|} \int_{E} v_{h}(\mathbf{x}) m_{\alpha}(\mathbf{x}) d x \quad \forall m_{\alpha} \in \mathcal{M}_{k-2}(E)
$$

Using these linear functionals the projection operator, $\Pi_{k}^{\nabla}: \tilde{\mathcal{V}}_{k}(E) \rightarrow \mathcal{P}_{k}(E)$, may be computed utilising the following orthogonality condition [67]:

$$
\left(\nabla p_{k}, \nabla\left(\Pi_{k}^{\nabla} v_{h}-v_{h}\right)\right)_{0, E}=0 \quad \forall \quad p_{k} \in \mathcal{P}_{k}(E)
$$

Equation 2.7 is rank-deficient as the gradients of constant polynomials are zero thus a projection onto constants $P_{0}: \tilde{\mathcal{V}}_{k}(E) \rightarrow \mathcal{P}_{0}(E)$ must be defined as a closure:

$$
P_{0} \Pi_{k}^{\nabla} v_{h}-P_{0} v_{h}=0 \quad \text { where } \quad P_{0} v_{h}=\int_{\partial E} v_{h} d s
$$

With the initial space $\tilde{\mathcal{V}}_{k}(E)$ and the projection $\Pi_{k}^{\nabla}$ defined it is now possible to introduce the enhanced local virtual element space $\mathcal{V}_{k}(E)$. This space is identical $\tilde{\mathcal{V}}_{k}(E)$ but possesses the additional property:

$$
\left(v_{h}, p_{k}\right)_{0, E}=\left(\Pi_{k}^{\nabla} v_{h}, p_{k}\right)_{0, E},
$$

where $v_{h} \in \tilde{\mathcal{V}}_{k}(E)$ and $p_{k} \in \tilde{\mathcal{P}}_{k-1}(E) \cup \tilde{\mathcal{P}}_{k}(E)$. $\tilde{\mathcal{P}}_{k}(E)$ is the set of polynomials of order strictly equal to $k$. Therefore, $\mathcal{V}_{k}(E)$ may be written formally as:

$$
\mathcal{V}_{k}(E)=\left\{v_{h} \in \tilde{\mathcal{V}}_{k}(E) \mid\left(v_{h}, p_{k}\right)_{0, E}=\left(\Pi_{k}^{\nabla} v_{h}, p_{k}\right)_{0, E} \quad \forall p_{k} \in \tilde{\mathcal{P}}_{k-1}(E) \cup \tilde{\mathcal{P}}_{k}(E)\right\}
$$


The linear functionals $\operatorname{dof}_{i}\left(v_{h}\right)$ defined on $\tilde{\mathcal{V}}_{k}(E)$ may be taken as the degrees of freedom of $\mathcal{V}_{k}(E)$ and given $\mathcal{V}_{k}(E) \subset \tilde{\mathcal{V}}_{k}(E)$ the projection $\Pi_{k}^{\nabla}$ is also well defined on $\mathcal{V}_{k}(E)$. The proof that this set of linear functionals comprise a unisolvent set of degrees of freedom is provided in [4]. The number of degrees of freedom on a polygon is therefore $N_{\text {dof }}=k N_{v}+n_{k-2}$. Each degree of freedom corresponds to a basis function, $\left\{\varphi_{i}\right\}_{i=1}^{N_{\text {dof }}}$, that span the space $\mathcal{V}_{k}(E)$ such that $\operatorname{dof}_{i}\left(\varphi_{j}\right)=\delta_{i j}$. Therefore, any $v_{h} \in \mathcal{V}_{k}(E)$ may be expanded in the VEM basis as follows [67]:

$$
v_{h}=\sum_{i=1}^{N_{\text {dof }}} \operatorname{dof}_{i}\left(v_{h}\right) \varphi_{i}
$$

It is important to note that $\mathcal{P}_{k}(E) \subset \mathcal{V}_{k}(E)$, it is precisely this property that guarantees the approximation properties of $\mathcal{V}_{k}(E)$. The local $L^{2}(E)$ projector $\Pi_{k}^{0}: \mathcal{V}_{k}(E) \rightarrow \mathcal{P}_{k}(E)$ is computable in $\mathcal{V}_{k}(E)$ using just the degrees of freedom and the following orthogonality condition:

$$
\left(p_{k}, \Pi_{k}^{0} v_{h}-v_{h}\right)_{0, E}=0 \quad \forall p_{k} \in \mathcal{P}_{k}(E) .
$$

Introducing the property of $\mathcal{V}_{k}(E)$ from equation 2.9 equation 2.12 becomes [67]:

$$
\left(p_{k}, \Pi_{k}^{0} v_{h}\right)_{0, E}=\left\{\begin{array}{l}
\left(p_{k}, v_{h}\right)_{0, E} \text { for } \quad p_{k} \in \mathcal{P}_{k-2}(E) \\
\left(p_{k}, \Pi_{k}^{\nabla} v_{h}\right)_{0, E} \quad \text { for } \quad p_{k} \in \tilde{\mathcal{P}}_{k-1}(E) \cup \tilde{\mathcal{P}}_{k}(E) .
\end{array}\right.
$$

The third and final projection operator required in this VEM formulation is the projection onto vector-valued polynomials $\Pi_{k-1}^{0} \nabla: \mathcal{V}_{k}(E) \rightarrow\left[\mathcal{P}_{k-1}(E)\right]^{2}$. This projection was introduced in [49] to deal with general second-order elliptic problems with heterogeneous coefficients. It was observed in [49] that the original projection $\Pi_{k}^{\nabla}$, when used to compute the diffusion operator, exhibited a loss of accuracy at higher orders when the diffusion matrix $\mathbf{D}(\mathbf{x}) \neq \mathbf{I}$. The projection $\Pi_{k-1}^{0} \nabla$ is computed using the following orthogonality condition:

$$
\left(\mathbf{p}_{k-1}, \Pi_{k-1}^{0} \nabla v_{h}-\nabla v_{h}\right)_{0, E}=0 \quad \forall \mathbf{p}_{k-1} \in\left[\mathcal{P}_{k-1}(E)\right]^{2}
$$

which gives:

$$
\left(\mathbf{p}_{k-1}, \Pi_{k-1}^{0} \nabla v_{h}\right)_{0, E}=\left(\mathbf{p}_{k-1}, \nabla v_{h}\right)_{0, E} .
$$

Integrating by parts the rhs of 2.15 gives:

$$
\left(\mathbf{p}_{k-1}, \nabla v_{h}\right)_{0, E}=\int_{\partial E} \mathbf{p}_{k-1} \cdot \mathbf{n} v_{h} d s-\int_{E} \nabla \cdot \mathbf{p}_{k-1} v_{h} d x
$$

where $\mathbf{n}$ is the outward unit normal to $\partial E$. This is computable from the degrees of freedom of $\mathcal{V}_{k}(E)$ as the integrand of the boundary term is a polynomial of order $2 k-1$ and the volume term may be computed from the internal degrees of freedom of $v_{h}$. The practical aspects of computing these projection operators (which are represented as matrices) are omitted here as they are covered in great detail in $[67,69]$. 


\subsection{The Global VEM Space}

This section will define the global virtual element space $\mathcal{V}_{k}(V)$ from which the global VEM solution is constructed [49]:

$$
\mathcal{V}_{k}(V)=\left\{v_{h} \in H^{1}(V):\left.v_{h}\right|_{E} \in \mathcal{V}_{k}(E) \quad \forall E \in \mathcal{T}_{h}\right\}
$$

The local variational forms of interest for a polygon $E$ are given below for $u_{h}, v_{h} \in \mathcal{V}_{k}(E)$ :

$$
\begin{gathered}
a^{E}\left(v_{h}, u_{h}\right)=\left(\mathbf{D}(\mathbf{x}) \nabla v_{h}, \nabla u_{h}\right)_{0, E}, \\
b^{E}\left(v_{h}, u_{h}\right)=\left(\Sigma(\mathbf{x}) v_{h}, u_{h}\right)_{0, E}, \\
f^{E}\left(v_{h}\right)=\left(f(\mathbf{x}), v_{h}\right)_{0, E},
\end{gathered}
$$

and the global variational forms are the usual FE assembly of their local counterparts [49]:

$$
\begin{aligned}
a\left(v_{h}, u_{h}\right) & =\sum_{E \in \mathcal{T}_{h}} a^{E}\left(v_{h}, u_{h}\right), \\
b\left(v_{h}, u_{h}\right) & =\sum_{E \in \mathcal{T}_{h}} b^{E}\left(v_{h}, u_{h}\right), \\
f\left(v_{h}\right) & =\sum_{E \in \mathcal{T}_{h}} f^{E}\left(v_{h}\right) .
\end{aligned}
$$

In VEM there is no way of directly computing functions $u_{h}, v_{h} \in \mathcal{V}_{k}(E)$ on the interior of the element therefore the following approximations are made to the continuous variational forms of equations 2.18-2.20 [49]:

$$
\begin{gathered}
a_{h}^{E}\left(v_{h}, u_{h}\right)=\left(\mathbf{D}(\mathbf{x}) \Pi_{k-1}^{0} \nabla v_{h}, \Pi_{k-1}^{0} \nabla u_{h}\right)_{0, E}+\bar{D} S_{E}^{\nabla}\left(\left(I-\Pi_{k}^{\nabla}\right) v_{h},\left(I-\Pi_{k}^{\nabla}\right) u_{h}\right), \\
b_{h}^{E}\left(v_{h}, u_{h}\right)=\left(\Sigma(\mathbf{x}) \Pi_{k}^{0} v_{h}, \Pi_{k}^{0} u_{h}\right)_{0, E}+\bar{\Sigma}|E| S_{E}^{0}\left(\left(I-\Pi_{k}^{0}\right) v_{h},\left(I-\Pi_{k}^{0}\right) u_{h}\right), \\
f_{h}^{E}\left(v_{h}\right)=\left(f(\mathbf{x}), \Pi_{k}^{0} v_{h}\right)_{0, E},
\end{gathered}
$$

where $\bar{D}$ and $\bar{\Sigma}$ are some constant approximations to $\mathbf{D}(\mathbf{x})$ and $\Sigma(\mathbf{x})$ inside $E$. The first term of $a_{h}^{E}\left(v_{h}, u_{u}\right)$ and $b_{h}^{E}\left(v_{h}, u_{u}\right)$ are the consistency terms. They ensure the $k$-consistency property is satisfied [4]:

$$
a_{h}^{E}(p, v)=a^{E}(p, v) \quad \text { and } \quad b_{h}^{E}(p, v)=b^{E}(p, v) \quad \forall v \in \mathcal{V}_{k}(E) \quad \text { and } \quad \forall p \in \mathcal{P}_{k}(E)
$$

The terms, $S_{E}^{\nabla}$ and $S_{E}^{0}$, ensure stability which means they enforce the following property [4]: 


$$
\begin{gathered}
\alpha_{*} a_{E}\left(v_{h}, v_{h}\right) \leq S_{E}^{\nabla}\left(v_{h}, v_{h}\right) \leq \alpha^{*} a_{E}\left(v_{h}, v_{h}\right), \\
\alpha_{*} b_{E}\left(w_{h}, w_{h}\right) \leq S_{E}^{0}\left(w_{h}, w_{h}\right) \leq \alpha^{*} b_{E}\left(w_{h}, w_{h}\right),
\end{gathered}
$$

where $v_{h}, w_{h} \in \mathcal{V}_{k}(E)$ such that $\Pi_{k}^{\nabla} v_{h}=0$ and $\Pi_{k}^{0} w_{h}=0$. Equations 2.28 and 2.29 mean that the stability terms have to scale like their respective bilinear forms on the kernel of their respective projections [49]. Satisfying equations 2.28-2.29 guarantees the stability of the method. In this paper the following choice of stabilisation was made [4]:

$$
\begin{aligned}
S_{E}^{\nabla}\left(\left(I-\Pi_{k}^{\nabla}\right) v_{h},\left(I-\Pi_{k}^{\nabla}\right) u_{h}\right) & =\sum_{r=1}^{N_{\text {dof }}} \operatorname{dof}_{r}\left(\left(I-\Pi_{k}^{\nabla}\right) v_{h}\right) \operatorname{dof}_{r}\left(\left(I-\Pi_{k}^{\nabla}\right) u_{h}\right), \\
S_{E}^{0}\left(\left(I-\Pi_{k}^{0}\right) v_{h},\left(I-\Pi_{k}^{0}\right) u_{h}\right) & =\sum_{r=1}^{N_{\text {dof }}} \operatorname{dof}_{r}\left(\left(I-\Pi_{k}^{0}\right) v_{h}\right) \operatorname{dof}_{r}\left(\left(I-\Pi_{k}^{0}\right) u_{h}\right) .
\end{aligned}
$$

There are other possible choices for the stabilisation, such as the so called "D-recipe", first introduced in [39], which at higher orders, and particularly for the 3D case, was shown to mitigate an observed degradation in the order of convergence. However, for the 2D cases presented in this paper the choices in equations 2.30 and 2.31 were sufficient. Again, if one wishes more information on how these discrete bilinear forms and stability terms (which in practice are matrices) are computed then there are a number of references that comprehensively discuss the implementation details $[67,69]$. That concludes this section on the VEM formulation, the next section will now present its application to the neutron diffusion equation.

\section{Discretisation of the Neutron Diffusion Equation}

\subsection{The Neutron Diffusion Equation (NDE)}

Let $V \subset \mathbb{R}^{2}$ be the domain of problem with boundary $\partial V$, which is the union of a vacuum boundary and reflective boundary $\Gamma_{V}$ and $\Gamma_{R}$ such that $\Gamma_{R} \cap \Gamma_{V}=\emptyset$. The steady-state, multigroup NDE is given below [13, 70]:

$$
\left\{\begin{array}{l}
-\nabla \cdot\left(D^{g}(\mathbf{x}) \nabla \phi_{g}(\mathbf{x})\right)+\Sigma_{r}^{g}(\mathbf{x}) \phi_{g}(\mathbf{x})=Q_{g}(\mathbf{x}) \quad \forall \mathbf{x} \in V \\
{\left[D^{g}(\mathbf{x}) \nabla \phi_{g}(\mathbf{x})\right] \cdot \mathbf{n}=0 \quad \forall \mathbf{x} \in \Gamma_{R},} \\
{\left[D^{g}(\mathbf{x}) \nabla \phi_{g}(\mathbf{x})\right] \cdot \mathbf{n}+\frac{1}{2} \phi_{g}(\mathbf{x})=0 \quad \forall \mathbf{x} \in \Gamma_{V}}
\end{array}\right.
$$

where $g=1, \ldots, G, D^{g}(\mathbf{x})$ is the group $g$ diffusion coefficient, $\Sigma_{r}^{g}(\mathbf{x})$ is the group $g$ macroscopic neutron removal cross-section, defined as:

$$
\Sigma_{r}^{g}(\mathbf{x})=\Sigma_{a}^{g}(\mathbf{x})+\sum_{g^{\prime} \neq g}^{G} \Sigma_{s}^{g \rightarrow g^{\prime}}(\mathbf{x})=\Sigma_{t}^{g}(\mathbf{x})-\Sigma_{s}^{g \rightarrow g}(\mathbf{x})
$$


where $\Sigma_{t}^{g}(\mathbf{x})$ is the macroscopic neutron total cross section. $Q_{g}(\mathbf{x})$ is a neutron source for group $g$ which can take many forms depending on the type of problem under consideration. This paper will only consider criticality problems where $Q_{g}(\mathbf{x})$ takes the form:

$$
Q_{g}(\mathbf{x})=\frac{\chi^{g}(\mathbf{x})}{\mathrm{K}_{\mathrm{eff}}} \sum_{g^{\prime}=1}^{G} \nu \Sigma_{f}^{g^{\prime}}(\mathbf{x}) \phi_{g^{\prime}}+\sum_{g^{\prime} \neq g}^{G} \Sigma_{s}^{g^{\prime} \rightarrow g}(\mathbf{x}) \phi_{g^{\prime}}(\mathbf{x})
$$

where $\chi_{g}(\mathbf{x})$ is the group $g$ prompt neutron fission spectrum, $\nu$ is the average number of neutrons per fission, $\Sigma_{f}^{g}(\mathbf{x})$ is the group $g$ fission cross section, $\Sigma_{s}^{g^{\prime} \rightarrow g}(\mathbf{x})$ is the scatter cross section from group $g^{\prime}$ to $g$ and $\mathrm{K}_{\mathrm{eff}}$ is the effective multiplication factor, an important quantity of interest (QoI) in nuclear reactor physics as it indicates the criticality of the system. By convention $g=1$ is the high energy (fast) flux and $g=G$ is the low energy (thermal) flux. Let $L_{g}=-\nabla \cdot D^{g} \nabla+\Sigma_{r}^{g}$ and $F_{g, g^{\prime}}=\chi_{g} \nu \Sigma_{f}^{g^{\prime}}$ then equation 3.1 is often written in matrix form $[1,70]$ :

$$
\left[\begin{array}{cccc}
L_{1} & 0 & 0 & \ldots \\
0 & L_{2} & 0 & \ldots \\
0 & 0 & L_{3} & \ldots \\
\vdots & \vdots & \vdots & \ldots
\end{array}\right]\left[\begin{array}{c}
\phi_{1} \\
\phi_{2} \\
\vdots \\
\phi_{G}
\end{array}\right]-\left[\begin{array}{cccc}
0 & \Sigma_{s}^{2 \rightarrow 1} & \Sigma_{s}^{3 \rightarrow 1} & \ldots \\
\Sigma_{s}^{1 \rightarrow 2} & 0 & \Sigma_{s}^{3 \rightarrow 2} & \ldots \\
\Sigma_{s}^{1 \rightarrow 3} & \Sigma_{s}^{2 \rightarrow 3} & 0 & \ldots \\
\vdots & \vdots & \vdots & \ldots
\end{array}\right]\left[\begin{array}{c}
\phi_{1} \\
\phi_{2} \\
\vdots \\
\phi_{G}
\end{array}\right]=\frac{1}{\gamma}\left[\begin{array}{cccc}
F_{1,1} & F_{1,2} & F_{1,3} & \ldots \\
F_{2,1} & F_{2,2} & F_{2,3} & \ldots \\
F_{3,1} & F_{3,2} & F_{3,3} & \ldots \\
\vdots & \vdots & \vdots & \ldots
\end{array}\right]\left[\begin{array}{c}
\phi_{1} \\
\phi_{2} \\
\vdots \\
\phi_{G}
\end{array}\right],
$$

or more succinctly in operator form:

$$
(L-S) \Phi=\frac{1}{\gamma} F \Phi \quad \text { where } \quad \Phi=\left[\phi_{1}, \ldots, \phi_{G}\right]^{T},
$$

from which it becomes clear that $\gamma$ are the eigenvalues of the operator $(L-S)^{-1} F$. It has been shown that spectrum of this operator form a positive decreasing series $\gamma_{1}>\gamma_{2}>\gamma_{3}>\ldots>0$ (see [71] chapter 3.3). The effective multiplication factor is defined as the first eigenvalue of the spectrum $K_{\text {eff }}=\gamma_{1}$. The corresponding eigenvector $\Phi_{1}$ is the fundamental mode of the system. Finding a discrete approximation to the the pair $\left(\Phi_{1}, \mathrm{~K}_{\text {eff }}\right)$ using the CG-VEM is the primary objective of the work contained in this paper.

\subsection{The Variational Formulation of the NDE}

Let $v=\left[v_{1}, \ldots, v_{G}\right]^{T}$ be a weight function, the variational formulation begins with multiplying equation 3.4 by $v^{T}$ and integrating over $V$ to give:

$$
\int_{V} v^{T} L \Phi d x-\int_{V} v^{T} S \Phi d x=\frac{1}{\gamma} \int_{V} v^{T} F \Phi d x
$$

Taking each term in turn beginning with the removal term. First integration by parts is applied to the diffusion term, followed by Gauss theorem together with the boundary conditions of equation 3.1 to give: 


$$
\begin{aligned}
\int_{V} v^{T} L \Phi d x & =\sum_{g=1}^{G} \int_{V} v_{g}\left(-\nabla \cdot D^{g} \nabla \phi_{g}\right) d x+\int_{V} v_{g} \Sigma_{r}^{g} \phi_{g} d x \\
& =\sum_{g=1}^{G} \int_{V} \nabla v_{g} \cdot D^{g} \nabla \phi_{g} d x+\int_{V} v_{g} \Sigma_{r}^{g} \phi_{g} d x+\frac{1}{2} \int_{\Gamma_{V}} v_{g} \phi_{g} d s
\end{aligned}
$$

from which the removal bilinear form, $\hat{L}(v, \Phi)$, is defined:

$$
\hat{L}(v, \Phi)=\sum_{g=1}^{G}\left(\nabla v_{g}, D^{g} \nabla \phi_{g}\right)_{0, V}+\left(v_{g}, \Sigma_{r}^{g} \phi_{g}\right)_{0, V}+\frac{1}{2}\left(v_{g}, \phi_{g}\right)_{0, \Gamma_{V}} .
$$

Next the scatter term:

$$
\int_{V} v^{T} S \Phi d x=\sum_{g=1}^{G} \sum_{g^{\prime} \neq g}^{G} \int_{V} v_{g} \Sigma_{s}^{g^{\prime} \rightarrow g} \phi_{g^{\prime}} d x
$$

from which the scatter bilinear form, $\hat{S}(v, \Phi)$, is defined:

$$
\hat{S}(v, \Phi)=\sum_{g=1}^{G} \sum_{g^{\prime} \neq g}^{G}\left(v_{g}, \Sigma_{s}^{g^{\prime} \rightarrow g} \phi_{g^{\prime}}\right)_{0, V}
$$

Finally, the fission term:

$$
\int_{V} v^{T} F \Phi=\sum_{g=1}^{G} \sum_{g^{\prime}=1}^{G} \int_{V} v_{g} \chi_{g} \nu \Sigma_{f}^{g^{\prime}} \phi_{g^{\prime}} d x
$$

from which the fission bilinear form, $\hat{F}(v, \Phi)$ is defined:

$$
\hat{F}(v, \Phi)=\sum_{g=1}^{G} \sum_{g^{\prime}=1}^{G}\left(v_{g}, \chi_{g} \nu \Sigma_{f}^{g^{\prime}} \phi_{g^{\prime}}\right)_{0, V}
$$

Note that the symbols for the bilinear forms $\hat{L}, \hat{S}$ and $\hat{F}$ have a circumflex above them to differentiate them from their operator counterparts $L, S$ and $F$. Using these bilinear forms we can formally pose the variational problem this paper will solve using CG-VEM:

$$
\left\{\begin{array}{l}
\text { Find } \lambda \in \mathbb{R} \text { such that there exists a } \Phi \in\left[H^{1}(V)\right]^{G} \text { which satisfies: } \\
\hat{L}(v, \Phi)-\hat{S}(v, \Phi)=\lambda \hat{F}(v, \Phi) \quad \forall v \in\left[H^{1}(V)\right]^{G}
\end{array} .\right.
$$

Given a polygonal decomposition of $V$, denoted $\mathcal{T}_{h}$, the following restrictions on the mesh, $\mathcal{T}_{h}$, must be made in addition to the ones stated in section 2.1:

1. The neutron diffusion coefficient and the macroscopic neutron cross-sections are all constant within each material sub-domain. For example within a nuclear fuel pin or the 
moderator.

2. In $\mathcal{T}_{h}$ there exists no polygon that crosses a material boundary. This means that every polygon must reside entirely within a material sub-domain and must conform to internal boundaries.

3. The set of edges in $\mathcal{T}_{h}$ that lie on the boundary $\partial V$ may be split into two disjoint sets of edges $\mathcal{E}_{R}$ and $\mathcal{E}_{V}$ that lie solely within $\Gamma_{R}$ and $\Gamma_{V}$ respectively. In other words, there exists no boundary edge that crosses the interface between $\Gamma_{R}$ and $\Gamma_{V}$.

Under these three assumptions integrals over $V$ may be split into the sum of integrals over all $E \in \mathcal{T}_{h}$ and constant material coefficients may be removed from each local integral. The values of the diffusion coefficient and cross sections within a given polygon are denoted by subscript $E, D_{E}^{g}, \Sigma_{r E}^{g}, \Sigma_{f E}^{g}$ and $\Sigma_{s E}^{g}$. To solve equation 3.9 on mesh $\mathcal{T}_{h}$ using CG-VEM discrete solutions are sought in the global VEM space $\mathcal{V}_{k}(E)$ (equation 2.17). Let:

$$
v^{h}=\left[v_{1}^{h}, \ldots, v_{G}^{h}\right]^{T} \in\left[\mathcal{V}_{k}(V)\right]^{G} \quad \text { and } \quad \Phi^{h}=\left[\phi_{1}^{h}, \ldots, \phi_{G}^{h}\right]^{T} \in\left[\mathcal{V}_{k}(V)\right]^{G},
$$

be weight and trial functions. Discrete bilinear forms, $\hat{L}^{h}\left(v^{h}, \Phi^{h}\right), \hat{S}^{h}\left(v^{h}, \Phi^{h}\right)$ and $\hat{F}^{h}\left(v^{h}, \Phi^{h}\right)$ that approximate their continuous counterparts, $\hat{L}\left(v^{h}, \Phi^{h}\right), \hat{S}\left(v^{h}, \Phi^{h}\right)$ and $\hat{F}\left(v^{h}, \Phi^{h}\right)$, are computed as follows:

$$
\begin{aligned}
& \hat{L}^{h}\left(v^{h}, \Phi^{h}\right)=\sum_{g=1}^{G} \sum_{E \in \mathcal{T}_{h}} a_{h}^{E}\left(v_{g}^{h}, \phi_{g}^{h}\right)+b_{h}^{E}\left(v_{g}^{h}, \phi_{g}^{h}\right)+\frac{1}{2} \sum_{e \in \mathcal{E}_{V}}\left(v_{g}^{h}, \phi_{g}^{h}\right)_{0, e}, \\
& \hat{S}^{h}\left(v^{h}, \Phi^{h}\right)=\sum_{g=1}^{G} \sum_{g^{\prime} \neq g}^{G} \sum_{E \in \mathcal{T}_{h}} \Sigma_{s E}^{g^{\prime} \rightarrow g}\left(\Pi_{k}^{0} v_{g}^{h}, \Pi_{k}^{0} \phi_{g^{\prime}}^{h}\right)_{0, E}, \\
& \hat{F}^{h}\left(v^{h}, \Phi^{h}\right)=\sum_{g=1}^{G} \sum_{g^{\prime}=1}^{G} \sum_{E \in \mathcal{T}_{h}} \chi_{g E} \nu \Sigma_{f E}^{g^{\prime}}\left(\Pi_{k}^{0} v_{g}^{h}, \Pi_{k}^{0} \phi_{g^{\prime}}^{h}\right)_{0, E},
\end{aligned}
$$

where:

$$
\begin{array}{r}
a_{h}^{E}\left(v_{g}^{h}, \phi_{g}^{h}\right)=D_{E}^{g}\left(\Pi_{k-1}^{0} \nabla v_{g}^{h}, \Pi_{k-1}^{0} \nabla \phi_{g}^{h}\right)_{0, E}+D_{E}^{g} S_{E}^{\nabla}\left(\left(I-\Pi_{k}^{\nabla}\right) v_{g}^{h},\left(I-\Pi_{k}^{\nabla}\right) \phi_{g}^{h}\right), \\
b_{h}^{E}\left(v_{g}^{h}, \phi_{g}^{h}\right)=\Sigma_{r E}^{g}\left(\Pi_{k}^{0} v_{g}^{h}, \Pi_{k}^{0} \phi_{g}^{h}\right)_{0, E}+\Sigma_{r E}^{g}|E| S_{E}^{0}\left(\left(I-\Pi_{k}^{0}\right) v_{g}^{h},\left(I-\Pi_{k}^{0}\right) \phi_{g}^{h}\right) .
\end{array}
$$

With the discrete bilinear forms defined, the discrete variational problem may be posed:

$$
\left\{\begin{array}{l}
\text { Find } \lambda^{h} \in \mathbb{R} \text { such that there exists a } \Phi^{h} \in\left[\mathcal{V}_{k}(V)\right]^{G} \text { which satisfies: } \\
\hat{L}^{h}\left(v^{h}, \Phi^{h}\right)-\hat{S}^{h}\left(v^{h}, \Phi^{h}\right)=\lambda^{h} \hat{F}^{h}\left(v^{h}, \Phi^{h}\right) \quad \forall v^{h} \in\left[\mathcal{V}_{k}(V)\right]^{G}
\end{array}\right.
$$

\subsection{Numerical Solution}

This section shall be primarily concerned with transforming equation 3.16 into matrix form such that a standard power iteration procedure may be performed to yield the fundamental eigenvalue $\lambda_{1}^{h}$ and fundamental mode $\Phi_{1}^{h}$. On each element, $E$, the weight and trial functions are expanded out in the local VEM basis: 


$$
\left.v_{g}^{h}\right|_{E}=\left.\sum_{i=1}^{N_{\text {dof }}^{E}} \operatorname{dof}_{i}\left(v_{g}^{h}\right) \varphi_{i} \quad \phi_{g}^{h}\right|_{E}=\sum_{i=1}^{N_{\text {dof }}^{E}} \operatorname{dof}_{i}\left(\phi_{g}^{h}\right) \varphi_{i},
$$

where $N_{\text {dof }}^{E}$ is the number of local degrees of freedom defined on element $E$, this value is the same for all energy groups. The local element matrices are derived by substituting the local expansions of equation 3.17 into the local bilinear forms in equations 3.14 and 3.15. Beginning with the diffusion bilinear form:

$$
\begin{aligned}
a_{h}^{E}\left(v_{g}^{h}, \phi_{g}^{h}\right) & =\sum_{i=1}^{N_{\text {dof }}^{E}} \sum_{j=1}^{N_{\text {dof }}^{E}} \operatorname{dof}_{i}\left(v_{g}^{h}\right) \operatorname{dof}_{j}\left(\phi_{g}^{h}\right) a_{h}^{E}\left(\varphi_{i}, \varphi_{j}\right) \\
& =\left(\mathbf{v}_{g}^{E}\right)^{T}\left(D_{E}^{g} \mathbf{K}_{E}+D_{E}^{g} \mathbf{S}_{E}^{\nabla}\right) \boldsymbol{\phi}_{g}^{E}
\end{aligned}
$$

where

$$
\left(\mathbf{K}_{E}\right)_{i, j}=\left(\Pi_{k-1}^{0} \nabla \varphi_{i}, \Pi_{k-1}^{0} \nabla \varphi_{j}\right)_{0, E} \quad\left(\mathbf{S}_{E}^{\nabla}\right)_{i, j}=S_{E}^{\nabla}\left(\left(I-\Pi_{k}^{\nabla}\right) \varphi_{i},\left(I-\Pi_{k}^{\nabla}\right) \varphi_{j}\right)
$$

are the stiffness consistency and stability matrices respectively. The vectors $\left(\mathbf{v}_{g}^{E}\right)_{i}=\operatorname{dof}_{i}\left(v_{g}^{h}\right)$ and $\left(\phi_{g}^{E}\right)_{i}=\operatorname{dof}_{i}\left(\phi_{g}^{h}\right)$ are the local degrees of freedom on element $E$ of $v_{g}^{h}$ and $\phi_{g}^{h}$ respectively. Next, the reaction bilinear form:

$$
\begin{aligned}
b_{h}^{E}\left(v_{g}^{h}, \phi_{g}^{h}\right) & =\sum_{i=1}^{N_{\text {dof }}^{E}} \sum_{j=1}^{N_{\text {dof }}^{E}} \operatorname{dof}_{i}\left(v_{g}^{h}\right) \operatorname{dof}_{j}\left(\phi_{g}^{h}\right) b_{h}^{E}\left(\varphi_{i}, \varphi_{j}\right) \\
& =\left(\mathbf{v}_{g}^{E}\right)^{T}\left(\Sigma_{r E}^{g} \mathbf{M}_{E}+\Sigma_{r E}^{g}|E| \mathbf{S}_{E}^{0}\right) \boldsymbol{\phi}_{g}^{E}
\end{aligned}
$$

where

$$
\left(\mathbf{M}_{E}\right)_{i, j}=\left(\Pi_{k}^{0} \varphi_{i}, \Pi_{k}^{0} \varphi_{j}\right)_{0, E} \quad\left(\mathbf{S}_{E}^{0}\right)_{i, j}=S_{E}^{0}\left(\left(I-\Pi_{k}^{0}\right) \varphi_{i},\left(I-\Pi_{k}^{0}\right) \varphi_{j}\right)
$$

are the mass consistency and stability matrices. A very similar process may be performed to the edges on the vacuum boundary to give:

$$
\left(v_{g}^{h}, \phi_{g}^{h}\right)_{0, e}=\left(\mathbf{v}_{g}^{e}\right)^{T} \mathbf{M}_{e} \phi_{g}^{e} \quad \text { where } \quad\left(\mathbf{M}_{e}\right)_{i, j}=\left(\varphi_{i}, \varphi_{j}\right)_{0, e}
$$

where $\mathbf{v}_{g}^{e}, \boldsymbol{\phi}_{g}^{e}$ are the vectors containing the local DoFs on edge $e$ of $v_{g}^{h}$ and $\phi_{g}^{h}$ respectively. Following the same argument for the local scatter and fission terms yields the following:

$$
\begin{gathered}
\sum_{s E}^{g^{\prime} \rightarrow g}\left(\Pi_{k}^{0} v_{g}^{h}, \Pi_{k}^{0} \phi_{g^{\prime}}^{h}\right)_{0, E}=\left(\mathbf{v}_{g}^{E}\right)^{T}\left(\Sigma_{s E}^{g^{\prime} \rightarrow g} \mathbf{M}_{E}\right) \boldsymbol{\phi}_{g}^{E} \\
\chi_{g E} \nu \Sigma_{f E}^{g^{\prime}}\left(\Pi_{k}^{0} v_{g}^{h}, \Pi_{k}^{0} \phi_{g^{\prime}}^{h}\right)_{0, E}=\left(\mathbf{v}_{g}^{E}\right)^{T}\left(\chi_{g E} \nu \Sigma_{f E}^{g^{\prime}} \mathbf{M}_{E}\right) \boldsymbol{\phi}_{g}^{E}
\end{gathered}
$$


With the local matrices defined (equations 3.19, 3.19, 3.24, 3.25 and 3.26) the derivation of the global system of equations may be explained. Let the vectors, $\mathbf{v}_{g}$ and $\boldsymbol{\phi}_{g}$, contain the global degrees of freedom of $v_{g}^{h}$ and $\phi_{g}^{h}$ over the entire mesh:

$$
\mathbf{v}_{g}=\left[\operatorname{dof}_{1}\left(v_{g}^{h}\right), \ldots, \operatorname{dof}_{N_{g}}\left(v_{g}^{h}\right)\right]^{T} \quad \phi_{g}=\left[\operatorname{dof}_{1}\left(\phi_{g}^{h}\right), \ldots, \operatorname{dof}_{N_{g}}\left(\phi_{g}^{h}\right)\right]
$$

where $N_{g}$ is the global number of degrees of freedom for the group $g$ flux. In principle each group scalar flux can be defined on a different mesh (of the same domain) and can therefore have a different number of degrees of freedom, see for example [12, 13, 22, 72]. However, the formulation presented in this paper shall be restricted to the case where all scalar fluxes are defined on the same mesh and therefore all have the same number of global degrees of freedom denoted $N$, therefore $N_{g}=N$ for all groups. Using the global DoF vectors the functions $v_{g}^{h}$ and $\phi_{g}^{h}$ may be expanded out in the full global basis as follows:

$$
v_{g}^{h}=\sum_{i=1}^{N}\left(\mathbf{v}_{g}\right)_{i} \varphi_{i} \quad \phi_{g}^{h}=\sum_{i=1}^{N}\left(\phi_{g}\right)_{i} \varphi_{i} .
$$

Note, that the local expansions in equation 3.17 are simply the restriction of the global expansions to those basis functions that are non-zero on a given element $E$. Substituting the local matrices into the expressions for the global bilinear forms (equations 3.11-3.13) yields the following global systems, beginning with the removal bilinear form:

$$
\begin{aligned}
\hat{L}^{h}\left(v^{h}, \Phi^{h}\right) & =\sum_{g=1}^{G}\left(\mathbf{v}_{g}\right)^{T}\left(\sum_{E \in \mathcal{T}_{h}} D_{E}^{g} \mathbf{K}_{E}+\Sigma_{r E}^{g} \mathbf{M}_{E}+D_{E}^{g} \mathbf{S}_{E}^{\nabla}+\Sigma_{r E}^{g}|E| \mathbf{S}_{E}^{0}+\frac{1}{2} \sum_{e \in \mathcal{E}_{V}} \mathbf{M}_{e}\right) \boldsymbol{\phi}_{g} \\
& =\sum_{g=1}^{G}\left(\mathbf{v}_{g}\right)^{T} \mathbf{L}_{g} \boldsymbol{\phi}_{g}
\end{aligned}
$$

where $\mathbf{L}_{g}$ is the global group $g$ removal operator, equal to the term inside the middle brackets of equation 3.29. Next, the scatter bilinear form:

$$
\begin{aligned}
\hat{S}^{h}\left(v^{h}, \phi^{h}\right) & =\sum_{g=1}^{G} \sum_{g^{\prime} \neq g}^{G}\left(\mathbf{v}_{g}\right)^{T}\left(\sum_{E \in \mathcal{T}_{h}} \Sigma_{s E}^{g^{\prime} \rightarrow g} \mathbf{M}_{E}\right) \boldsymbol{\phi}_{g^{\prime}} \\
& =\sum_{g=1}^{G} \sum_{g^{\prime}=1}^{G}\left(\mathbf{v}_{g}\right)^{T} \mathbf{S}_{g, g^{\prime}} \boldsymbol{\phi}_{g^{\prime}}
\end{aligned}
$$

and finally the fission bilinear form 


$$
\begin{aligned}
\hat{F}^{h}\left(v^{h}, \phi^{h}\right) & =\sum_{g=1}^{G} \sum_{g^{\prime}=1}^{G}\left(\mathbf{v}_{g}\right)^{T}\left(\sum_{E \in \mathcal{T}_{h}} \chi_{g E} \nu \Sigma_{f E}^{g^{\prime}} \mathbf{M}_{E}\right) \boldsymbol{\phi}_{g^{\prime}} \\
& =\sum_{g=1}^{G} \sum_{g^{\prime}=1}^{G}\left(\mathbf{v}_{g}\right)^{T} \mathbf{F}_{g, g^{\prime}} \boldsymbol{\phi}_{g^{\prime}}
\end{aligned}
$$

Substituting each of these global matrices into equation 3.16 gives:

$$
\sum_{g=1}^{G}\left(\mathbf{v}_{g}\right)^{T}\left(\mathbf{L}_{g} \phi_{g}-\sum_{g^{\prime} \neq g}^{G} \mathbf{S}_{g, g^{\prime}} \boldsymbol{\phi}_{g^{\prime}}-\frac{1}{\lambda} \sum_{g^{\prime}=1}^{G} \mathbf{F}_{g, g^{\prime}} \boldsymbol{\phi}_{g^{\prime}}\right)=0
$$

Because each weight function $v_{g}^{h} \in \mathcal{V}_{k}(V)$ is arbitrary then the term inside the brackets of equation 3.35 must be zero for every group $g$. Therefore, equation 3.35 may be rewritten as the following global block system of equations:

$$
\left[\begin{array}{cccc}
\mathbf{L}_{1} & \mathbf{0} & \mathbf{0} & \ldots \\
\mathbf{0} & \mathbf{L}_{2} & \mathbf{0} & \ldots \\
\mathbf{0} & \mathbf{0} & \mathbf{L}_{3} & \ldots \\
\vdots & \vdots & \vdots & \ldots
\end{array}\right]\left[\begin{array}{c}
\boldsymbol{\phi}_{1} \\
\boldsymbol{\phi}_{2} \\
\vdots \\
\boldsymbol{\phi}_{G}
\end{array}\right]-\left[\begin{array}{cccc}
\mathbf{0} & \mathbf{S}_{1,2} & \mathbf{S}_{1,3} & \ldots \\
\mathbf{S}_{2,1} & \mathbf{0} & \mathbf{S}_{2,3} & \ldots \\
\mathbf{S}_{3,1} & \mathbf{S}_{3,2} & \mathbf{0} & \ldots \\
\vdots & \vdots & \vdots & \ldots
\end{array}\right]\left[\begin{array}{c}
\boldsymbol{\phi}_{1} \\
\boldsymbol{\phi}_{2} \\
\vdots \\
\boldsymbol{\phi}_{G}
\end{array}\right]=\frac{1}{\lambda}\left[\begin{array}{cccc}
\mathbf{F}_{1,1} & \mathbf{F}_{1,2} & \mathbf{F}_{1,3} & \ldots \\
\mathbf{F}_{2,1} & \mathbf{F}_{2,2} & \mathbf{F}_{2,3} & \ldots \\
\mathbf{F}_{3,1} & \mathbf{F}_{3,2} & \mathbf{F}_{3,3} & \ldots \\
\vdots & \vdots & \vdots & \ldots
\end{array}\right]\left[\begin{array}{c}
\boldsymbol{\phi}_{1} \\
\boldsymbol{\phi}_{2} \\
\vdots \\
\boldsymbol{\phi}_{G}
\end{array}\right]
$$

With the matrix form of the variational problem defined the power iteration procedure may be described. Let $\boldsymbol{\phi}_{g}^{(i)}$ denote the $i$ 'th iterate of the group $g$ global flux vector and $\mathrm{K}_{\mathrm{eff}}^{(i)}$ the $i$ 'th iterate of the effective multiplication factor. The power iteration begins by initialising the fluxes to some initial flux profile, in this case unit neutron scalar flux $\boldsymbol{\phi}_{g}^{(0)}=\mathbf{1}$ and $\mathrm{K}_{\mathrm{eff}}^{(0)}=1$. The iterative procedure then follows equation 3.36 below [70]:

$$
\boldsymbol{\phi}_{g}^{(i+1)}=\mathbf{L}_{g}^{-1}\left(\frac{1}{K_{\mathrm{eff}}^{(i)}} \sum_{g^{\prime}=1}^{G} \mathbf{F}_{g g^{\prime}} \boldsymbol{\phi}_{g^{\prime}}^{(i)}+\sum_{g^{\prime}<g}^{G} \mathbf{S}_{g g^{\prime}} \boldsymbol{\phi}_{g^{\prime}}^{(i+1)}+\sum_{g^{\prime}>g}^{G} \mathbf{S}_{g g^{\prime}} \boldsymbol{\phi}_{g^{\prime}}^{(i)}\right)
$$

After each full power iteration a new set of fluxes will have been computed:

$$
\left\{\phi_{1}^{(i+1)}, \phi_{2}^{(i+1)}, \ldots, \phi_{G}^{(i+1)}\right\}
$$

The discrete global fluxes for each iteration, denoted $\phi_{g}^{h,(i)}$ are computed from the global DoF vectors using the global expansion given in equation 3.28. After each power iteration the next iterate of the effective multiplication factor $\mathrm{K}_{\mathrm{eff}}^{(i+1)}$ may be computed as follows

$$
\mathrm{K}_{\mathrm{eff}}^{(i+1)}=\mathrm{K}_{\mathrm{eff}}^{(i)} \frac{\int_{V} S_{f}^{(i+1)} d x}{\int_{V} S_{f}^{(i)} d x} \quad \text { where } \quad S_{f}^{(i)}=\sum_{g^{\prime}=1}^{G} \nu \Sigma_{f}^{g^{\prime}} \phi_{g}^{h,(i)}
$$

The computation of these fission integrals requires some explanation as the process for $k=1$ differs from that for $k>1$. This is because for the higher orders the integrals of fluxes may be 
computed from the first internal degree of freedom on each element. While, for the case $k=1$, there are no internal degrees of freedom so the integral must be computed by other means. The iteration superscript has been omitted here for clarity.

$$
\int_{V} \sum_{g^{\prime}=1}^{G} \nu \Sigma_{f}^{g^{\prime}} \phi_{g^{\prime}}^{h}=\sum_{g^{\prime}=1}^{G} \sum_{E \in \mathcal{T}_{h}} \nu \Sigma_{f E}^{g^{\prime}} \int_{E} \phi_{g^{\prime}}^{h} d x .
$$

Then, each flux integral in element $E$ is computed as follows:

$$
\int_{E} \phi_{g^{\prime}}^{h} d x=\left\{\begin{array}{rll}
\int_{E} \Pi_{k}^{0} \phi_{g^{\prime}}^{h} d x & \text { if } & k=1 \\
|E| \operatorname{dof}_{k N_{v}+1}\left(\phi_{g^{\prime}}^{h}\right) & \text { if } & k>1
\end{array} .\right.
$$

The iteration procedure is continued until a chosen convergence criteria is satisfied, in this case the following criteria was used:

$$
\max _{g} \delta_{g}<\epsilon \quad \text { where } \quad \delta_{g}=\frac{\left\|\phi_{g}^{(i+1)}-\boldsymbol{\phi}_{g}^{(i)}\right\|_{2}}{\left\|\boldsymbol{\phi}_{g}^{(i)}\right\|_{2}}
$$

$\|\cdot\|_{2}$ denotes the Euclidean norm and $\epsilon$ is some tolerance chosen based on the problem. The value of $\epsilon$ varied for each problem and so will be omitted here and stated in their respective sections. The group removal operators $\mathbf{L}_{g}$ were symmetric positive definite (SPD) and therefore could be solved using the preconditioned conjugate gradient method (PCG) with the HYPRE BoomerAMG preconditioner. All of the large-scale linear algebra was implemented using PETSc [73] and parallelised using MPI. The convergence criteria for the PCG solver was altered throughout the power iteration in order to improve the rate of convergence. Given a linear system $\mathbf{A x}=\mathbf{b}$ then for for PCG iteration $i$ the residual is given by $\mathbf{r}_{i}=\mathbf{b}-\mathbf{A} \mathbf{x}_{i}$. The PCG iterations are continued until the following convergence criteria are met [73]:

$$
\left\|\mathbf{r}_{i}\right\|_{2}<\max \left(r_{\text {tol }}\|\mathbf{b}\|_{2}, a_{\text {tol }}\right)
$$

The absolute tolerance, $a_{\text {tol }}$, was held constant at the PETSc default value of $1 \times 10^{-50}$. The value of the relative tolerance $r_{\text {tol }}$ was varied as the power iteration progressed. Each removal operator matrix $\mathbf{L}_{g}$ had its own relative tolerance, denoted $r_{\text {tol }}^{g}$, which was computed as follows:

$$
r_{\mathrm{tol}}^{g}=\min \left(0.1 \delta_{g}, 5 \times 10^{-3}\right)
$$

where $\delta_{g}$ is given in equation 3.41. Therefore, $r_{\text {tol }}^{g}$ is capped at a maximum value of $5 \times 10^{-3}$ and decreases as the relative difference in flux iterates decreases, thus providing a more accurate solution as the power iteration approaches convergence. Varying $r_{\text {tol }}^{g}$ throughout the power iteration did not effect the final solution and would greatly improve the rate of convergence; especially for the higher VEM orders where the number of PCG iterations can grow large. Some numerical examples will be presented in section 4.1 demonstrating the convergence behaviour of the various VEM orders on both Cartesian and polygonal meshes. The expression for $r_{\text {tol }}^{g}$ given in equation 3.43 was simply a heuristic based on trial and error, not the result of a rigorous attempt to find the largest admissible $r_{\text {tol }}^{g}$. Upon completion of the power iteration the neutron 
scalar flux is typically normalised such that the fission source is unity:

$$
\frac{1}{\mathrm{~K}_{\mathrm{eff}}} \int_{V} \sum_{g=1}^{g} \nu \Sigma_{f}^{g}(\mathbf{x}) \phi_{g}(\mathbf{x}) d x=1 .
$$

However, the choice of neutron scalar flux normalisation is arbitrary and different benchmark problems will often specify different normalisation's.

\subsection{Convergence Behaviour}

This section will discuss how the convergence behaviour of both the power iteration and the PCG solves vary with VEM order $k$ and the type of mesh used (i.e. Cartesian or polygonal). Some numerical examples will also be given in section 4.1. The three factors that determine the length of time required to converge a power method are:

- The computational cost of performing a matrix-vector multiply (MVM) with global matrices. This is an operation that is performed both within the PCG algorithm itself to compute the action of the $\mathbf{L}_{g}^{-1}$ operator (see [74], chapter 6.7 for a description of the PCG algorithm), and in computing the fission and scatter sources in equation 3.36.

- The number of iterations required to converge PCG solve for each $\mathbf{L}_{g}$. This quantity depends on the spectral radii the operators $\mathbf{L}_{g}$, which in turn depend on the VEM order $k$ and the mesh type.

- The number of iterations required to converge the power iteration. This is a property of the optical thickness of the system. If the system is highly diffusive with a lot of scatter relative to absorption then the power method may converge arbitrarily slowly [1].

Only the first two items shall be discussed here as they are the only two significantly affected by the VEM order and the type of mesh used. The effect of VEM order and mesh type on the third item is marginal. Typically slow convergence of the power iteration is solved by use of acceleration techniques such as diffusion synthetic acceleration. However, such techniques are beyond the scope of this paper. Starting with the number of global degrees of freedom, in a mesh $\mathcal{T}_{h}$ with $N_{v}$ vertices, $N_{e}$ edges and $N_{f}$ faces the number of global DoFs is given by

$$
N=N_{v}+(k-1) N_{e}+n_{k-2} N_{f}
$$

where $n_{k-2}=k(k-1) / 2$ (equation 2.3) which is the same for all elements. Therefore the number of degrees of freedom scales quadratically with $k$ and linearly with the number of vertices, edges and faces. The number of global degrees of freedom is important as it determines size of the global system of equations, a key factor in the computational cost of a MVM. Another factor that determines the length of a matrix-vector multiply is the number of non-zero (NNZ) values per row in the global matrix. To see how this quantity scales with VEM order consider figure 2. The global degree of freedom $\operatorname{dof}_{i}\left(v_{h}\right)$ is a vertex degree of freedom, which is shared by three faces. It is therefore coupled to all of the degrees of freedom of all three faces. For $k=1$, $\operatorname{dof}_{i}\left(v_{h}\right)$ is coupled to 13 other DoFs including itself so there will be 13 non-zero values on row $i$ of all of the global matrices $\mathbf{L}_{g}$. For $k=3 \operatorname{dof}_{i}\left(v_{h}\right)$ is coupled to 13 vertex DoFs, 30 edge DoFs and 9 face DoFs for a total of 52 non-zeros on row $i$. The number of non-zeros per row is highly variable for polygonal meshes because the number of edges per element is highly variable. While elements in Cartesian meshes, by definition, always have four sides, so this quantity is both smaller on average and more predictable. 

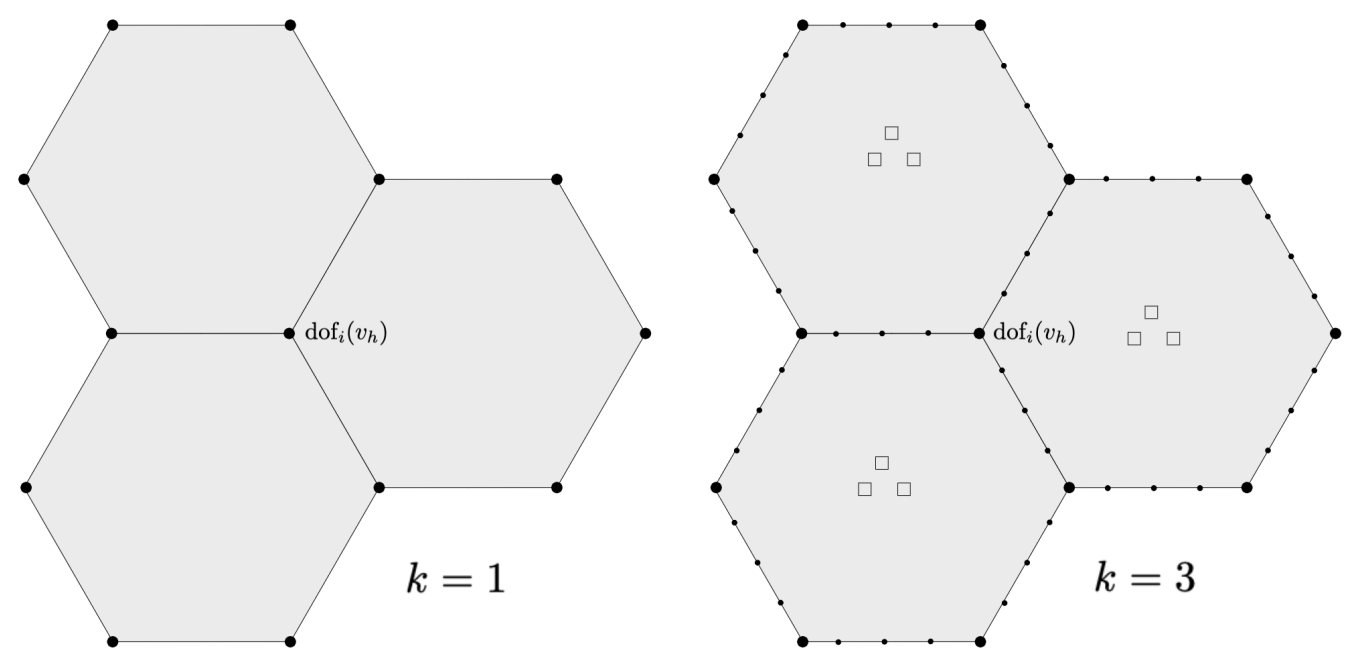

Figure 2: Distribution of DoFs on three elements that all share a vertex.

The condition number of the removal operators $\mathbf{L}_{g}$ grows as function of the VEM order $k$. One measure of condition number, the spectral condition number $\rho=\lambda_{\max } / \lambda_{\min }$, is directly related to the rate of convergence for the PCG method. Given a linear system $\mathbf{A x}=\mathbf{b}$ with exact solution $\mathbf{x}^{*}$ and $i$ 'th iteration $\mathbf{x}_{i}$ the following error bound holds [74]:

$$
\left\|\mathbf{x}^{*}-\mathbf{x}_{i}\right\|_{\mathbf{A}} \leq 2\left[\frac{\sqrt{\rho}-1}{\sqrt{\rho}+1}\right]^{i}\left\|\mathbf{x}^{*}-\mathbf{x}_{0}\right\|_{\mathbf{A}}=2 C(\rho, i)\left\|\mathbf{x}^{*}-\mathbf{x}_{0}\right\|_{\mathbf{A}}
$$

where $\|\mathbf{x}\|_{\mathbf{A}}=(\mathbf{A x}, \mathbf{x})$. As $\rho \rightarrow \infty$ then $C(\rho, i) \rightarrow 1$ and the PCG solver will converge arbitrarily slowly. So, as expected, numerical schemes involving higher VEM orders will require more PCG iterations and will therefore take longer to converge each PCG solve. With regards to mesh type: whether polygonal meshes produce global matrices with higher condition numbers compared to block-Cartesian meshes depends on the geometry of the problem under consideration. Block-Cartesian meshes, such as those for the UO2 pincell in section 4.2.1, can exhibit distortion and poor aspect ratio depending on the geometric mappings used to create them. Under these conditions, the condition numbers of the removal operators may increase substantially compared to an ideal rectilinear Cartesian mesh. Meanwhile, the major source of ill-conditioning for centroidal Voronoi meshes is the presence of small edges relative to the characteristic element diameter. However, this problem may be solved by deleting edges that fall below a given tolerance and by performing many Lloyds iterations to smooth the centroidal Voronoi diagram when generating the mesh $[75,76]$. That concludes the discussion on the application of CG-VEM to the NDE. The next section shall present two nuclear reactor benchmarks to demonstrate the application of the VEM to the NDE. 


\section{Results}

This section shall demonstrate the use of CG-VEM to solve the two-dimensional (2D) IAEA and C5G7 nuclear reactor physics verification benchmark problems. These are well-known verification benchmarks and are often used determine the accuracy, and verify the implementation, of nuclear reactor physics software. In addition to the numerical examples presented in this section, two method of manufactured solutions (MMS) verification benchmark test cases are presented in the appendix. The purpose of the MMS test cases are to demonstrate that the CG-VEM presented in this paper converges with the theoretical order of accuracy.

\subsection{D IAEA Nuclear Reactor Physics Verification Benchmark Problem}

The 2D IAEA benchmark problem, specified in page 437 of [77], is a two group LWR problem developed to test nuclear reactor physics software by the Argonne National Lab (ANL) in 1977. A schematic of the geometry is presented below in figure 3. The numbered square grid

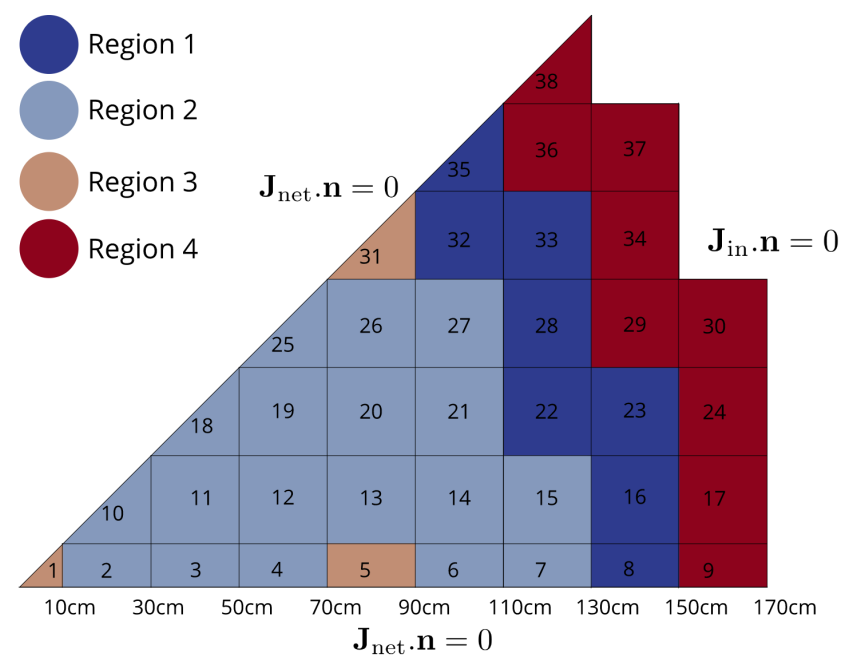

Figure 3: The 2D IAEA problem geometry.

represents the sub-assemblies of the problem in which sub-assembly powers are computed. The symbol $V$ refers to the full domain while $V_{c}$ refers to the core, which is the union of regions 1, 2 and 3 shown in figure 3. The problem utilises symmetry to reduce the problem to a $1 / 8$ core. Therefore, the two internal boundaries (bottom and diagonal boundaries) have prescribed reflective boundary conditions while the external boundary has a prescribed vacuum condition. The reflective and vacuum boundaries are denoted $\Gamma_{R}$ and $\Gamma_{V}$ respectively. This verification benchmark test case utilises a buckling approximation within the 2D neutron diffusion equation to account for axial (z-direction) neutron leakage. The resulting partial differential equations (PDEs) and prescribed boundary conditions are given by

$$
\left\{\begin{array}{l}
-\nabla \cdot\left(D_{1}(\mathbf{x}) \nabla \phi_{1}(\mathbf{x})\right)+\left(\Sigma_{a}^{1}(\mathbf{x})+\Sigma_{s}^{1 \rightarrow 2}(\mathbf{x})+D_{1}(\mathbf{x}) B_{z}^{2}\right) \phi_{1}(\mathbf{x})=\frac{1}{\mathrm{~K}_{\mathrm{eff}}} \nu \Sigma_{f}^{2}(\mathbf{x}) \phi_{2}(\mathbf{x}) \quad \forall \mathbf{x} \in V \\
-\nabla \cdot\left(D_{2}(\mathbf{x}) \nabla \phi_{2}(\mathbf{x})\right)+\left(\Sigma_{a}^{2}(\mathbf{x})+D_{2}(\mathbf{x}) B_{z}^{2}\right) \phi_{2}(\mathbf{x})=\Sigma_{s}^{1 \rightarrow 2}(\mathbf{x}) \phi_{1}(\mathbf{x}) \quad \forall \mathbf{x} \in V \\
-D_{g} \nabla \phi_{g} \cdot \mathbf{n}=\frac{1}{2} \cdot\left(\frac{1-\beta}{1+\beta}\right) \phi_{g} \quad \forall \mathbf{x} \in \Gamma_{V}
\end{array}\right.
$$


The axial buckling is $B_{z}^{2}=0.8 \times 10^{-4} \mathrm{~cm}^{-2}$ and the IAEA specification states that the vacuum boundary be implemented as an albedo boundary with a very small albedo, in this case $\beta=0.0317787$. The IAEA specification also states that the neutron scalar fluxes should be normalised such that:

$$
\frac{1}{\left|V_{c}\right|} \int_{V_{c}} \sum_{g=1}^{G} \nu \Sigma_{f}^{g}(\mathbf{x}) \phi_{g}(\mathbf{x}) d x=1 .
$$

The macroscopic neutron cross sections for this problem are given below in table 1

\begin{tabular}{c|cccccc|c} 
Region & $D_{1}$ & $D_{2}$ & $\Sigma_{s}^{1 \rightarrow 2}$ & $\Sigma_{a}^{1}$ & $\Sigma_{a}^{2}$ & $\nu \Sigma_{f}^{2}$ & Material \\
\hline 1 & 1.5 & 0.4 & 0.02 & 0.01 & 0.08 & 0.135 & Fuel 1 \\
2 & 1.5 & 0.4 & 0.02 & 0.01 & 0.085 & 0.135 & Fuel 2 \\
3 & 1.5 & 0.4 & 0.02 & 0.01 & 0.13 & 0.135 & Fuel 2 + Rod \\
4 & 2.0 & 0.3 & 0.04 & 0 & 0.01 & 0 & Reflector
\end{tabular}

Table 1: Nuclear data for the 2D IAEA problem, taken from [77].

All other macroscopic neutron cross-sections not given in table 1 are assumed to be zero. The QoIs in this benchmark are as follows:

- The effective multiplication factor $\mathrm{K}_{\mathrm{eff}}$

- The group neutron scalar flux integrals over the whole domain:

$$
\mathrm{I}_{1}=\int_{V} \phi_{1}(\mathbf{x}) d x \quad \mathrm{I}_{2}=\int_{V} \phi_{2}(\mathbf{x}) d x
$$

- The average sub-assembly powers:

$$
\mathrm{P}_{i}=\frac{1}{\left|V_{i}\right|} \int_{V_{i}} \sum_{g=1} \nu \Sigma_{f}^{g}(\mathbf{x}) \phi_{g}(\mathbf{x}) d x
$$

where $V_{i}$ is the volume of sub-assembly $i$. This QoI is calculated only for sub-assemblies 23 and 28 (see figure 3), these particular powers were chosen because they lie at the interface of the fuel and moderator and therefore will exhibit larger errors than in the interior of the core due to larger flux gradients within those regions. Therefore, they provide a better measure of the quality of the solution.

These QoIs are different to the ones specified in [77]. These changes were made to reduce the number of results to a smaller set for the purpose of clarity. The CG-VEM reference case was a Cartesian mesh with 48,330 elements run with $k=4$. This mesh contained 629,651 spatial degrees of freedom per group. As well as the reference CG-VEM solution, an isogeometric analysis (IGA) neutron diffusion and transport code called ICARUS (Isogeometric Continuous self-Adjoint Radiation Using Splines) was utilised as a means of providing an additional highfidelity benchmark verification solution. The ICARUS code, and its associated isogeometric spatial discretisation, is described in detail elsewhere [23, 24]. The IGA simulation was performed with $k=4$ and had 87,648 DoFs per group. The results of both the CG-VEM reference case and the IGA case are given below in table 2 . The results of the CG-VEM reference case and the IGA case are in close agreement. Additionally, the flux maps for the CG-VEM reference solution solution are shown in figure 4. 


\begin{tabular}{|c|c|c|}
\hline QoI & CG-VEM reference case & IGA case \\
\hline $\mathrm{K}_{\mathrm{eff}}$ & 1.02958867 & 1.02958867 \\
$\mathrm{I}_{1}$ & $2.90264549 \mathrm{e}+05$ & $2.90264548 \mathrm{e}+05$ \\
$\mathrm{I}_{2}$ & $8.83396422 \mathrm{e}+04$ & $8.83396464 \mathrm{e}+04$ \\
$\mathrm{P}_{23}$ & $6.28005258 \mathrm{e}-01$ & $6.28005213 \mathrm{e}-01$ \\
$\mathrm{P}_{28}$ & $1.52337123 \mathrm{e}+00$ & $1.52337068 \mathrm{e}+00$ \\
\hline
\end{tabular}

Table 2: The values of the QoIs for the CG-VEM reference case and the IGA case.
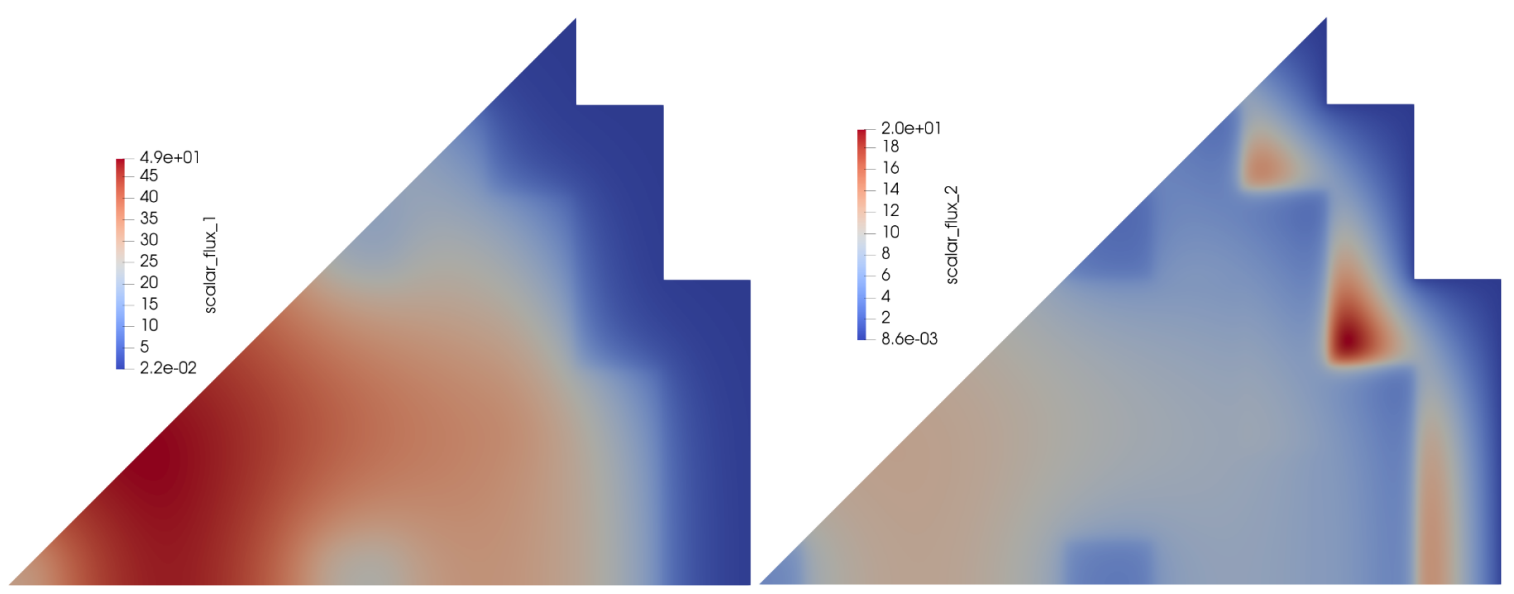

Figure 4: The neutron scalar flux contours (fast flux left thermal flux right) for the reference IAEA solution.

Two types of meshes were generated for this problem: a Cartesian mesh and a polygonal mesh. An example of each are given in figure 5 .
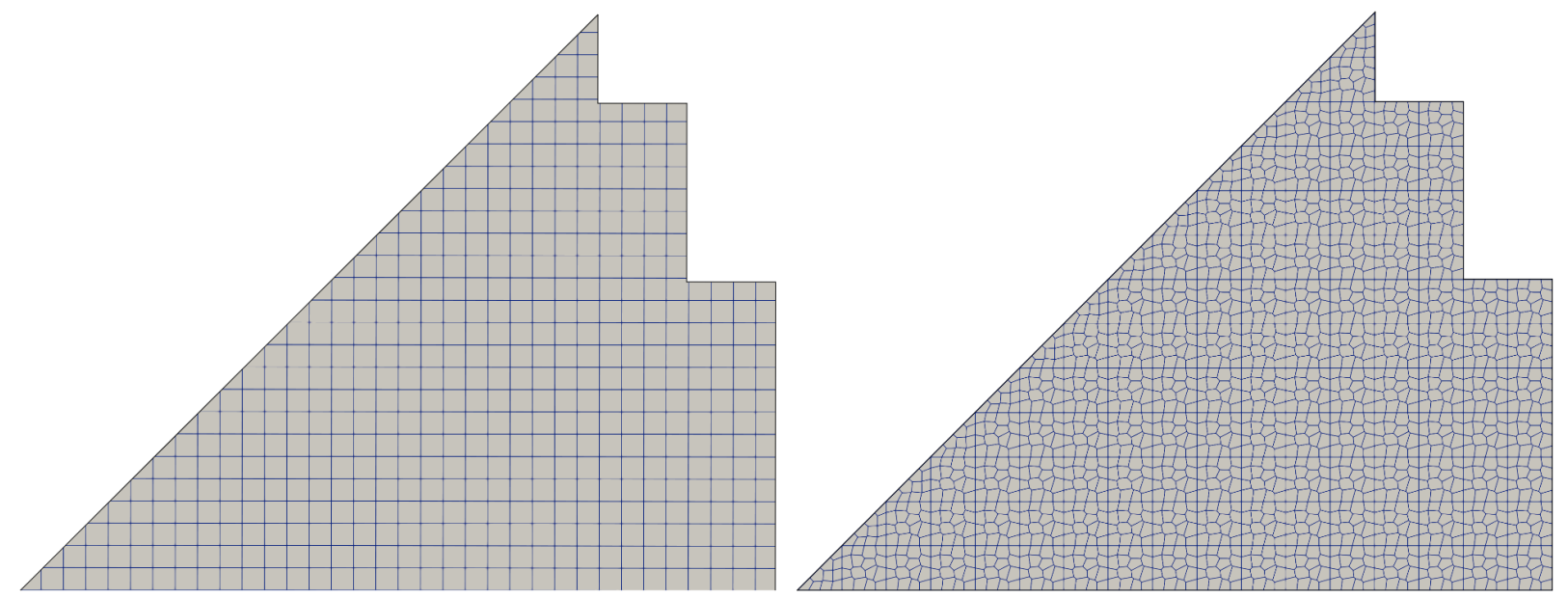

Figure 5: A Cartesian mesh of the IAEA-2D problem comprised of 495 elements and polygonal mesh comprised of 1954 elements.

It's important to note that the Cartesian meshes for this problem are ideal, rectilinear Cartesian meshes with no distortion or poor aspect ratio. One would therefore expect the best matrix conditioning and the lowest errors for these meshes. This is useful as it means the numerical effect of using a polygonal mesh may be observed. The IAEA-2D problem was solved using VEM orders $k=1,2,3,4$ for both the Cartesian meshes and the polygonal meshes. The power iteration tolerance (the convergence criteria detailed in equation 3.41) was set at $1 \times 10^{-14}$. At this tolerance between 620-650 power iterations were needed to reach convergence, with slightly more power iterations needed for more refined meshes and higher orders. There was 
negligible difference between Cartesian and polygonal meshes with regards to the number of power iterations required. However, as expected there were large increases in the number of PCG iterations needed to converge each inner solve as VEM order increased. All values of error in the QoIs presented in figures 6 and 7 are with respect the CG-VEM reference case values in table 2. These figures plot both the Cartesian and the polygonal mesh results on the same graph to facilitate easy comparison between the two.

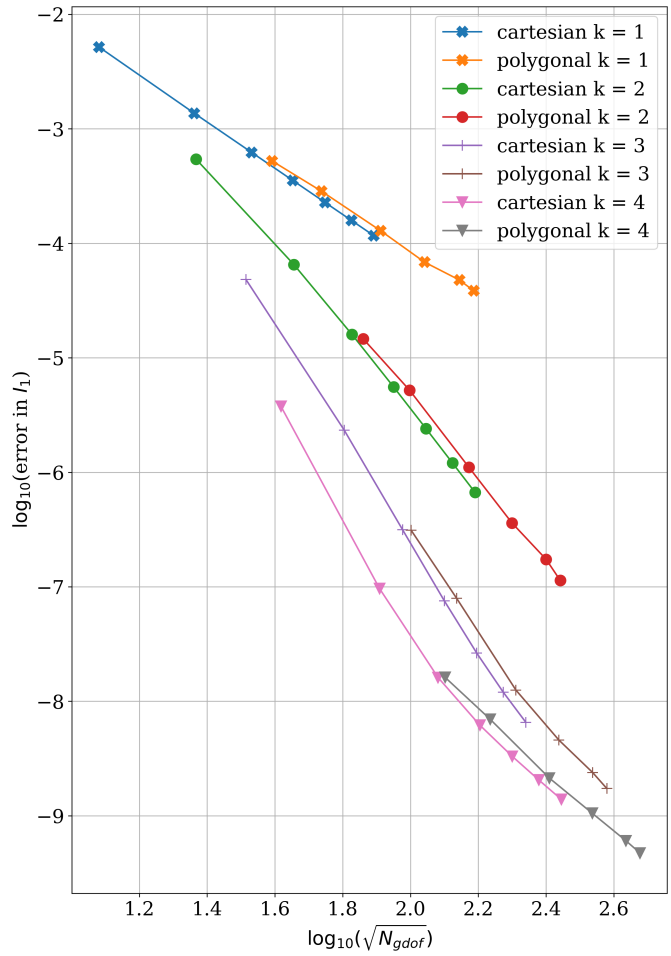

(a) Relative error in $\mathrm{I}_{1}$.

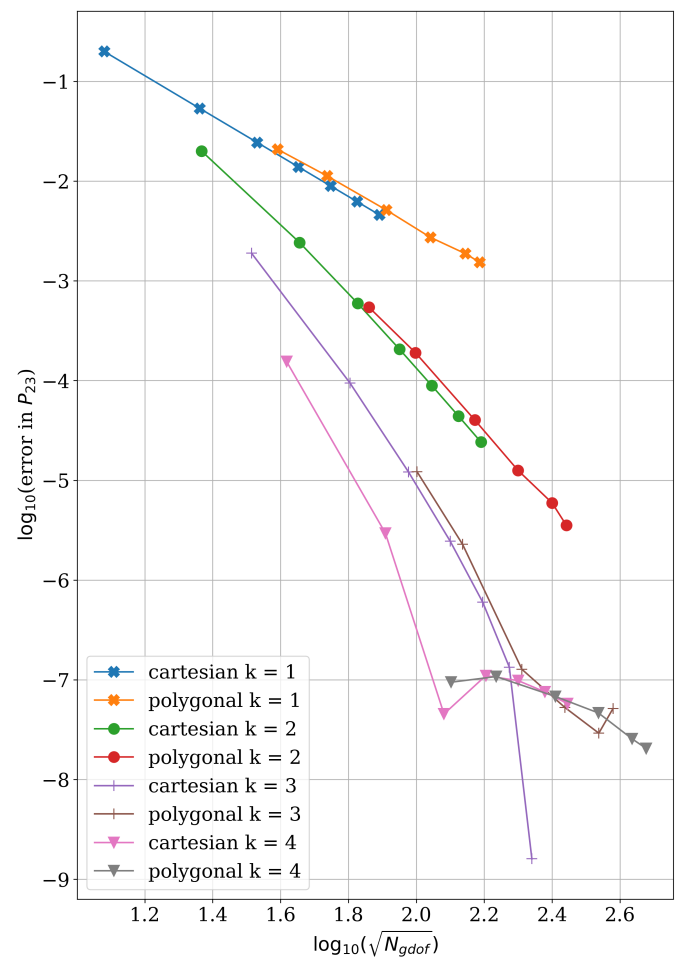

(c) Relative error in $\mathrm{P}_{23}$.

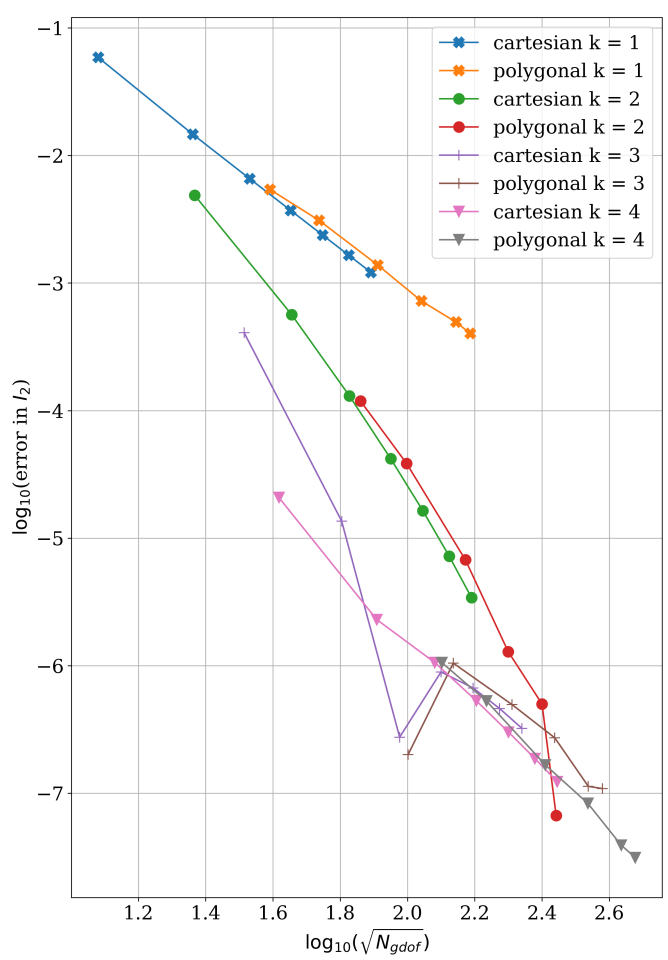

(b) Relative error in $\mathrm{I}_{2}$.

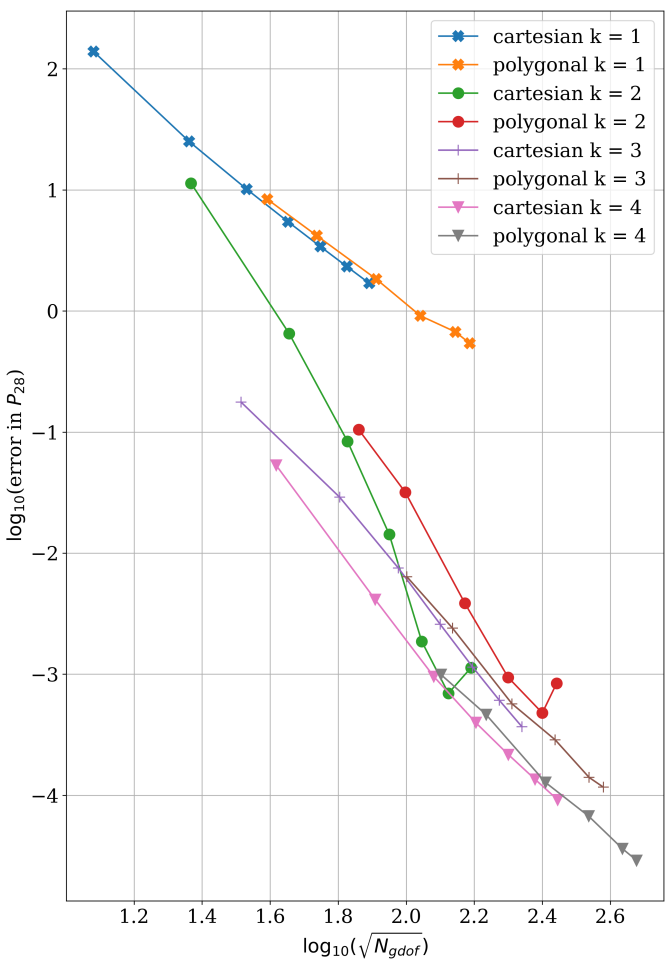

(d) Relative error in $\mathrm{P}_{28}$.

Figure 6: The errors in $\mathrm{I}_{1}, \mathrm{I}_{2}, \mathrm{P}_{23}$ and $\mathrm{P}_{28}$ for both the Cartesian and polygonal meshes with respect to the CG-VEM reference, table 2. 


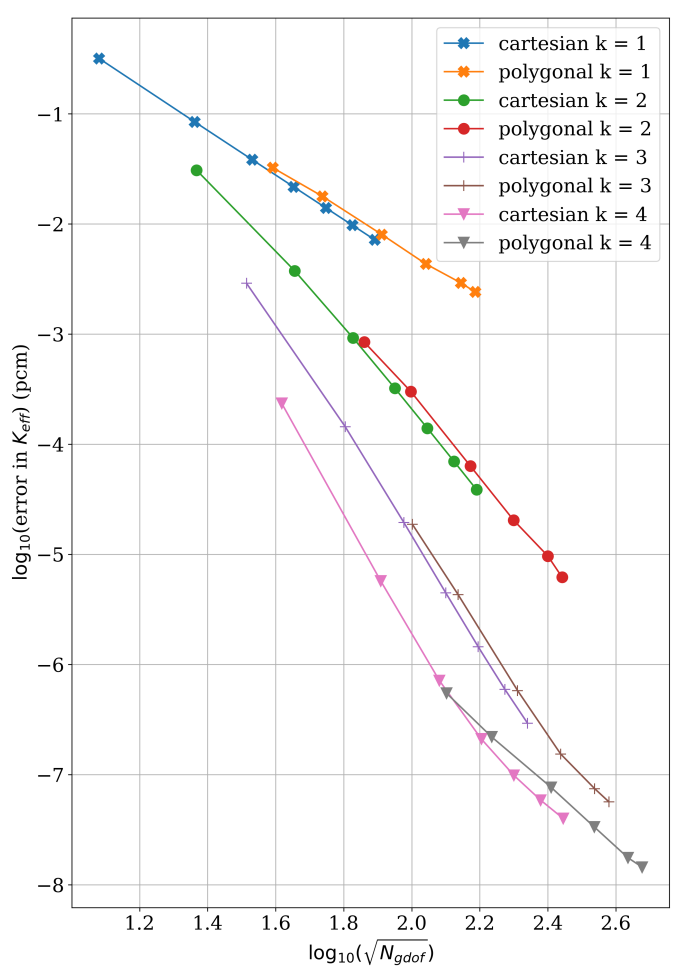

Figure 7: Absolute error in pcm of $\mathrm{K}_{\text {eff }}$ for both the Cartesian and polygonal meshes with respect to the CG-VEM reference, table 2.

Figures 6 and 7 show a number of things. First, that the errors in the QoIs become small, which means that the results are in good agreement with the CG-VEM solution. Second, that as the VEM order is increased the error curves shift downward and become steeper. This means that increasing the order both reduces the error but also increases the rate convergence toward the CG-VEM reference solution. Third, that there does not appear to be much difference between the Cartesian and polygonal error curves. This means that the use of polygonal meshes, as opposed to the perfectly rectilinear ones, did not introduce any significant errors into the solution. However, it is important to note that the polygonal meshes used here were generated using a centroidal Voronoi tessellation with many (around 100) Lloyds iterations (see [75] for a description of Lloyds iteration). This meant that they were highly regular with regard to element diameter and edge length. These two factors greatly affect the conditioning of the global removal matrices $\mathbf{L}_{g}$. A topic of future work could be to investigate how the errors in the QoIs behave as the quality of polygonal mesh is decreased. It should be stressed that the benefit of using polygonal meshes is not its numerical performance relative to Cartesian or block-Cartesian meshes but rather the ease of meshing highly complex domains with general topology [61, 62, 63, 64, 65].

In addition to the numerical results given in figures 6 and 7 some results concerning the topics of section 3.4 shall be given here. Beginning with how the number of global degrees of freedom $N$ and number of matrix non-zeros $N_{\mathrm{nz}}$ scale with the number of virtual elements in a mesh. Figures $8 \mathrm{a}$ and $8 \mathrm{~b}$ show that both $N$ and $N_{\mathrm{nz}}$ grow linearly with the number of elements with the constants of proportionality increasing with order $k$. Additionally they show both $N$ and $N_{\mathrm{nz}}$ grow faster for polygonal meshes than for Cartesian meshes. As was explained in section 3.4, this is because polygonal meshes have elements with many more edges on average. Finally, in figure 9 the total computational time required for the power iterations to converge, denoted $T_{\mathrm{PI}}$ is plotted against the number of degrees of freedom of the global system for both the Cartesian and polygonal IAEA meshes. The simulations were run on an HP linux workstation with an Intel Xeon E5-2687W v3 @ 3.10GHz processor. This has been done to determine 
whether polygonal meshes take longer to converge than the Cartesian meshes when controlling for both VEM order and the number of global DoFs

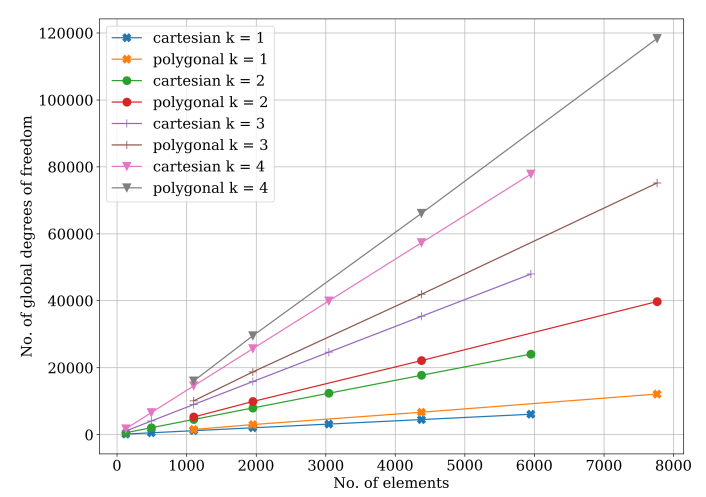

(a) $N$ versus the no. of elements.

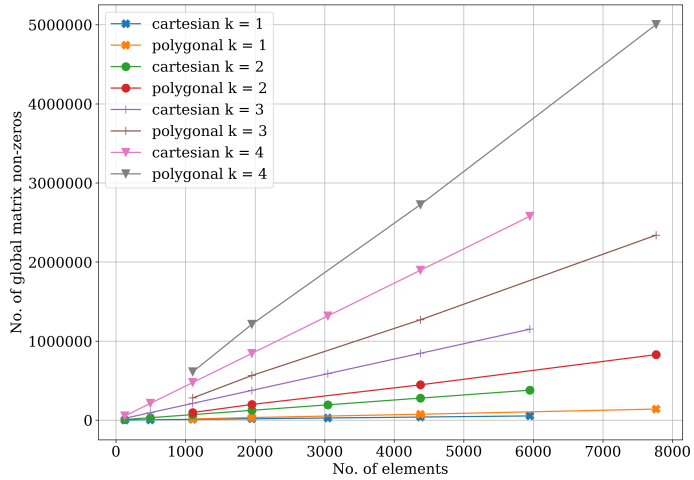

(b) $N_{\text {nz }}$ versus the no. of elements.

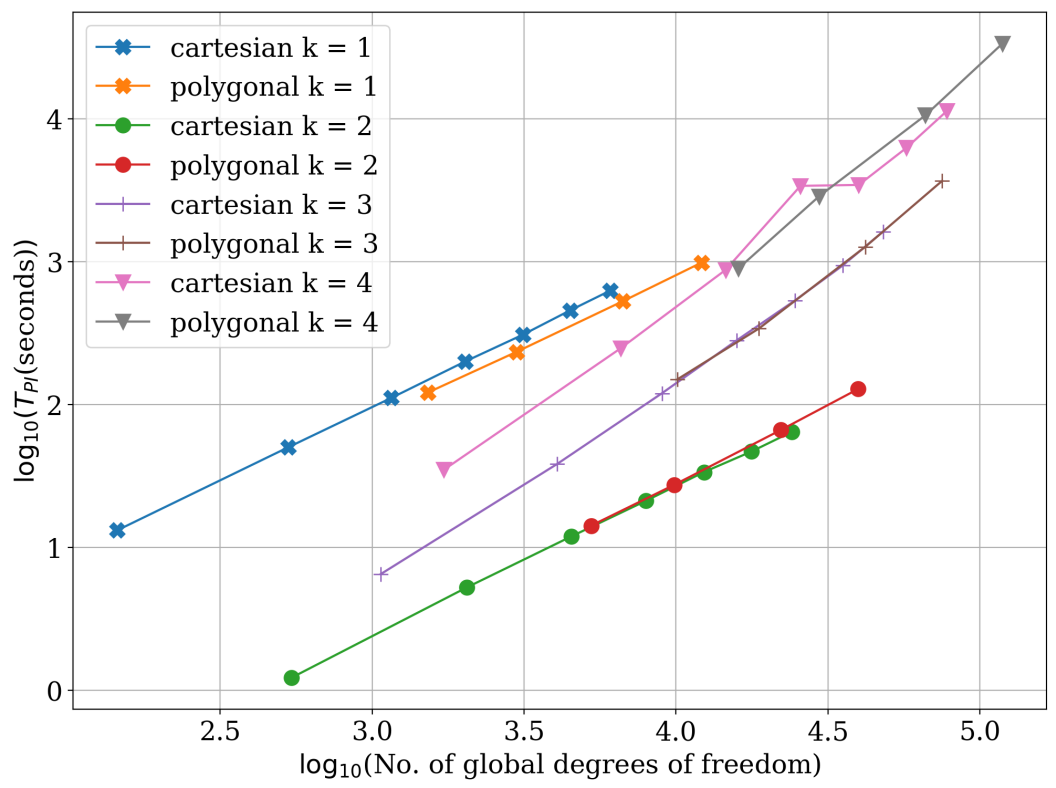

Figure 9: The time taken to converge the power iteration $\log _{10}\left(T_{\mathrm{PI}}\right)$ as a function of $\log _{10}(N)$.

Figure 9 shows that there is not an appreciable difference in power iteration CPU execution time for the polygonal meshes compared to the Cartesian when controlling for $k$ and $N$. The curves for each order almost overlap. However, at a given number of global DoFs the polygonal mesh will have fewer elements compared to the Cartesian. Again, it must be stressed that the polygonal meshes used here were highly regular and that poor-quality polygonal meshes are unlikely to perform as well due to the resulting ill-conditioning. The reason the $k=1$ curves in figure 9 are shifted above the other orders is because the fission integral takes longer to compute when there are no internal degrees of freedom (see equation 3.40). That concludes this section on the IAEA problem, the next section shall now present results for the C5G7 nuclear reactor physics verification benchmark test case. 


\subsection{The 2D OECD-NEA C5G7 Nuclear Reactor Physics Verification Benchmark Problem}

The two-dimensional (2D) C5G7 benchmark, specified in [78], is a series of four adjacent MOX and UOX nuclear fuel assemblies (a checkerboard or colour-set configuration) with a water reflector verification benchmark problem. It is designed to test the ability of neutron diffusion and transport codes to solve realistic nuclear reactor physics problems that have not been spatially homogenised. A schematic of the 2D quarter-core (1/4) configuration is presented below in figure 10:
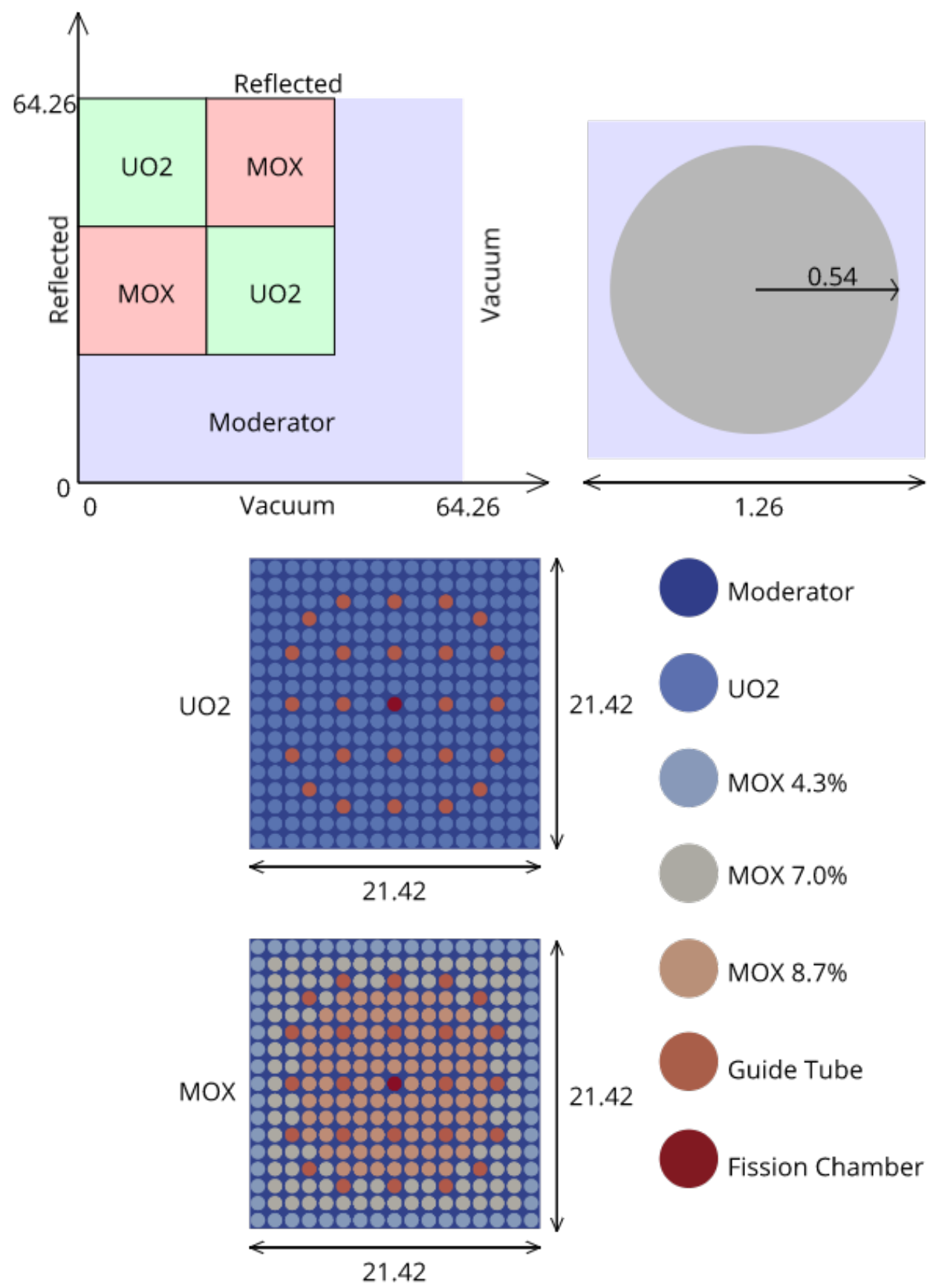

Figure 10: The C5G7 nuclear fuel assembly geometry and nuclear fuel pin schematic. All dimensions are in cm.

Each sub-assembly is a 17-by-17 array of nuclear fuel pins with the dimensions shown in figure 10. The macroscopic neutron cross-sections for each of these materials is given in [78]. As opposed to the IAEA-2D benchmark presented in section 4.1 the C5G7 geometry has contains circular fuel pins. This means a component of the error in the VEM solution results from the 
discrepancy between the mesh, which is composed entirely of straight edges, and the exact curved geometry of the nuclear fuel pincells within the C5G7 benchmark. To demonstrate the effect of this geometric discrepancy on the convergence rates of integral quantities a UO2 nuclear fuel pincell problem is presented. This problem, because of its simplicity relative to the full C5G7 benchmark, is a good test case to isolate the effect of the geometric error. After the pincell test case the remainder of this section presents the results of the full C5G7 benchmark.

\subsubsection{UO2 Nuclear Fuel Pincell Problem}

This test case presents a quarter $(1 / 4)$ symmetry nuclear fuel pincell with the dimensions shown in figure 10. The macroscopic neutron cross-sections of the C5G7 UO2 fuel were applied to the nuclear fuel pin and the cross-sections of the C5G7 moderator applied to the region surrounding the nuclear fuel pin. This test case was performed with VEM element orders $k=1,2,3,4$ and with a series of 8 semi-structured meshes containing between 45 and 500 VEM elements.
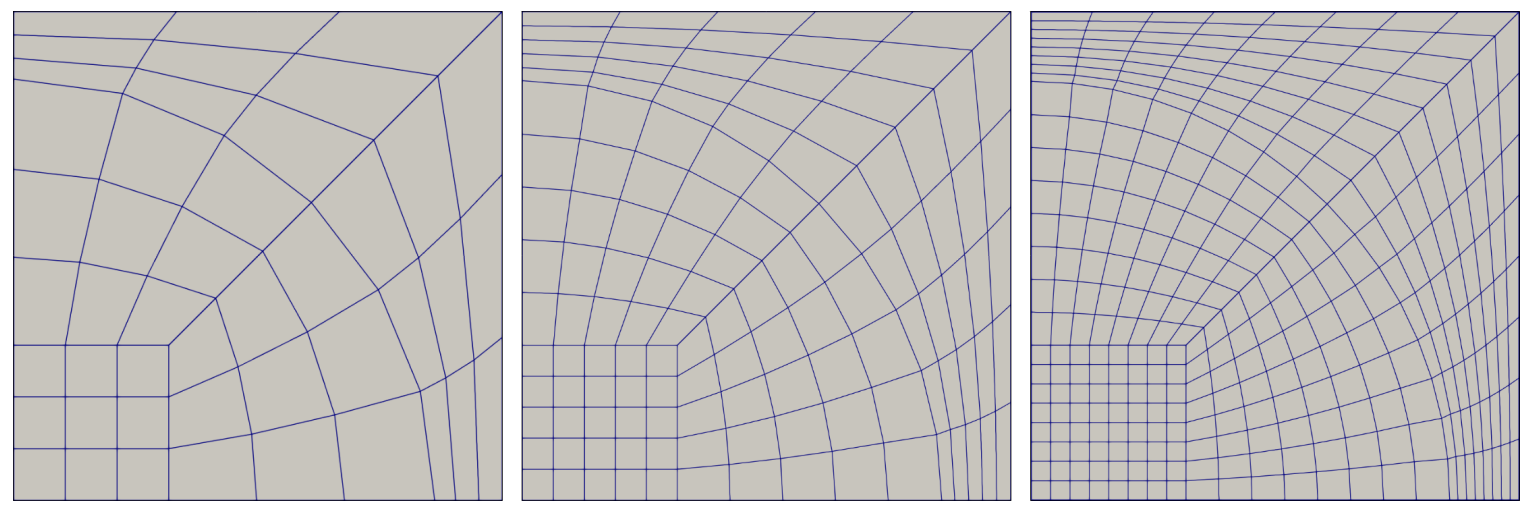

Figure 11: The pincell meshes with 45, 125 and 320 elements.

The vertices that lie on the interface between the nuclear fuel pin and the surrounding moderator were displaced outward radially to enforce conservation of fissile mass relative to the continuous geometry. This introduced errors in the arc length of the interface which in turn introduced errors into the CG-VEM solution. The discretisation error in the arc length compared to the true geometric arc length induces errors in the leakage of neutrons between the nuclear fuel pin and moderator. At higher orders this geometric error becomes the dominant error in the solution resulting in no further accuracy being gained from order elevation. This is demonstrated in the convergence curves in figure 12 where the order of convergence in the $k=3,4 \mathrm{CG}$-VEM schemes appear to be limited to that of the 2nd order scheme. The same effect was observed in [21] for the same pincell problem where FE method was compared to IGA. The FE solutions, because they too approximated the curved geometry with straight edges, saw the higher order convergence rates saturate at the 2 nd order convergence rate. To demonstrate the same effect in CG-VEM the following QoIs were selected:

1. The Integral of the group 1 and group 7 neutron scalar flux in the fuel

$$
I_{1}^{f}=\int_{V_{f}} \phi_{1}(\mathbf{x}) d x \quad I_{7}^{f}=\int_{V_{f}} \phi_{7}(\mathbf{x}) d x .
$$

2. The integral of the group 1 and group 7 neutron scalar flux in the moderator

$$
I_{1}^{m}=\int_{V_{m}} \phi_{1}(\mathbf{x}) d x \quad I_{7}^{m}=\int_{V_{m}} \phi_{7}(\mathbf{x}) d x .
$$


3. The effective multiplication factor $\mathrm{K}_{\mathrm{eff}}$

The reference case was run using VEM order $k=4$ and with 1125 elements, the results from which are tabulated in table 3

\begin{tabular}{|c|c|c|c|c|c|}
\hline QoI & $I_{1}^{f}$ & $I_{7}^{f}$ & $I_{1}^{m}$ & $I_{7}^{m}$ & $\mathrm{~K}_{\text {eff }}$ \\
\hline Value & 4.37792 & 1.44871 & 3.20068 & 1.13457 & 1.32600 \\
\hline
\end{tabular}

Table 3: The values of the QoIs for the reference case.

The power iteration convergece tolerance (equation 3.41) for this problem was set to $1 \times 10^{-15}$. All the errors in the QoI convergence plots (figures 12a to 12e) are are with respect to the values given in table 3 .

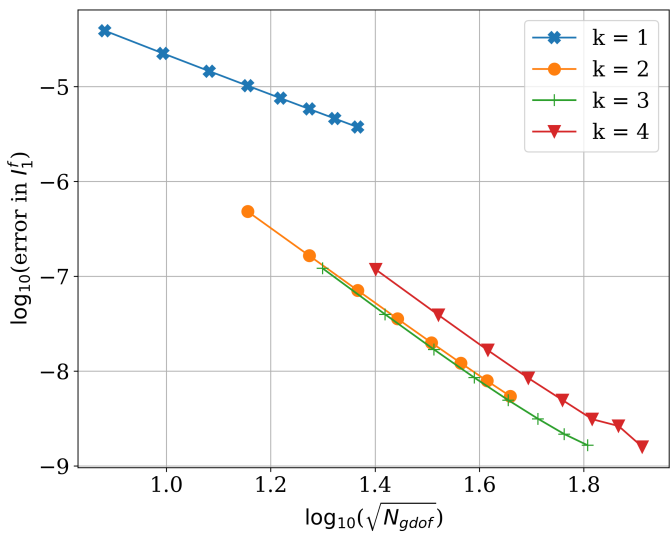

(a) Relative error in $I_{1}^{f}$.

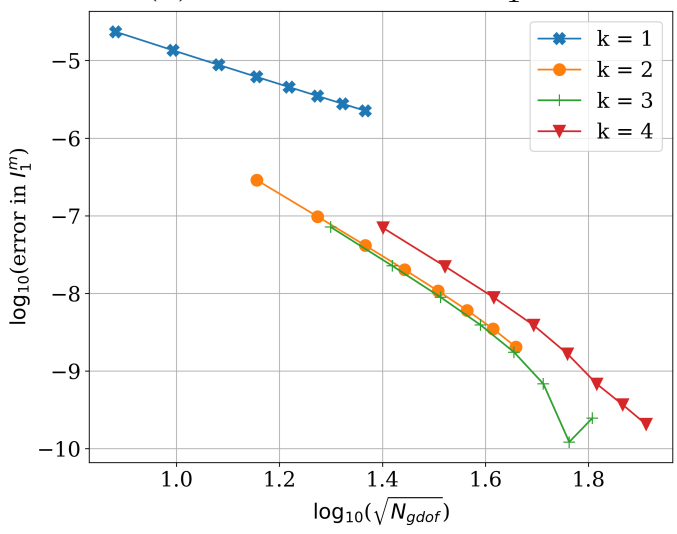

(c) Relative error in $I_{1}^{m}$.

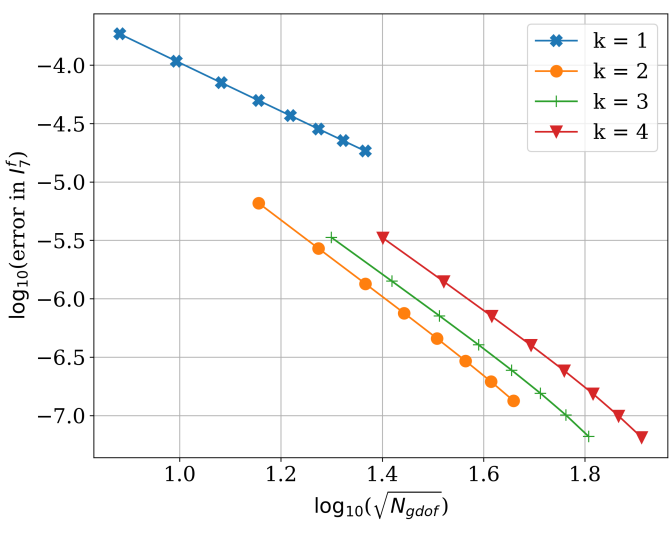

(b) Relative error in $I_{7}^{f}$.

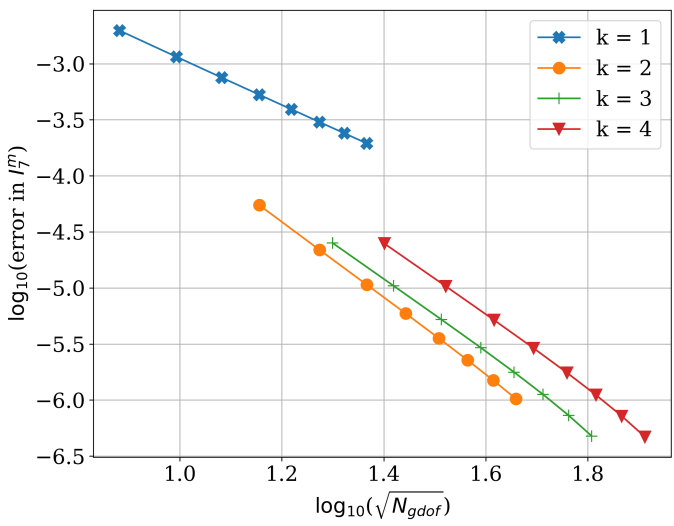

(d) Relative error in $I_{7}^{m}$.

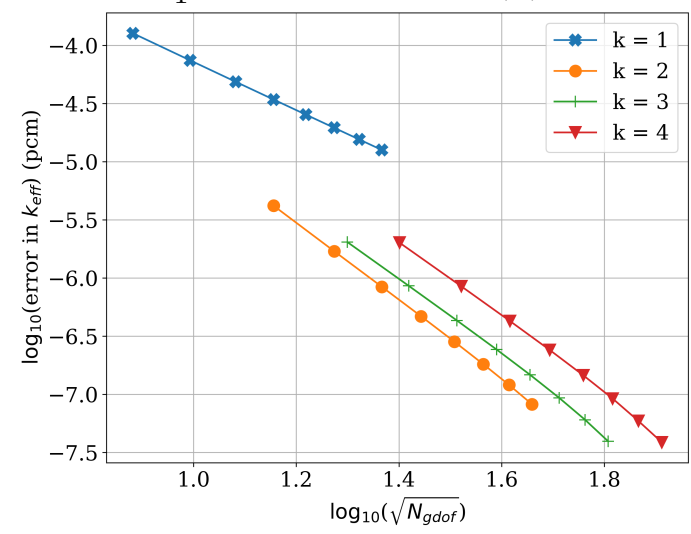

(e) Relative error in $\mathrm{K}_{\text {eff. }}$.

Figure 12: The errors in the QoIs relative to the values in table 3. 
It is clear from the convergence curves that increasing the order above $k=2$ does not appear to improve the order of convergence of any of the integral quantities or the $K_{\text {eff. }}$ To further test this hypothesis the same problem was performed using ICARUS [23, 24], a code based upon the exact-geometry IGA spatial discretisation method, yielding the following results shown in figure 13:

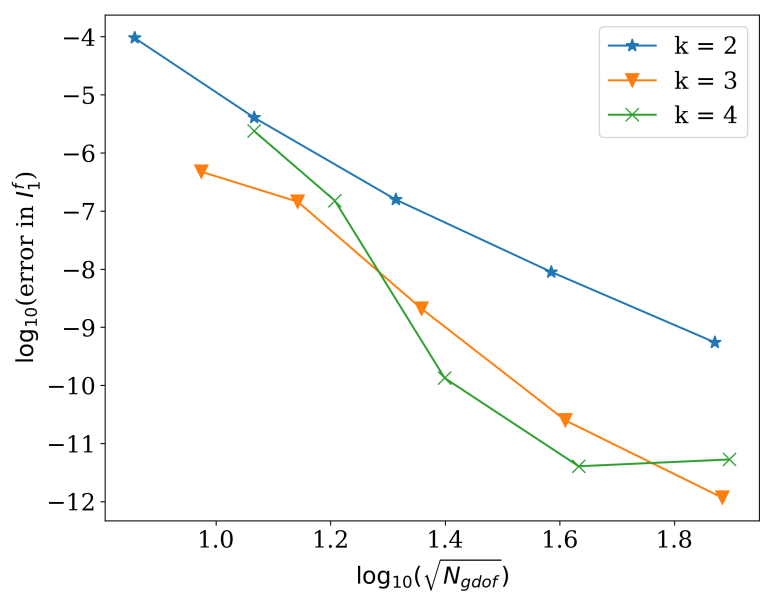

(a) Relative error in $I_{1}^{f}$ from IGA code

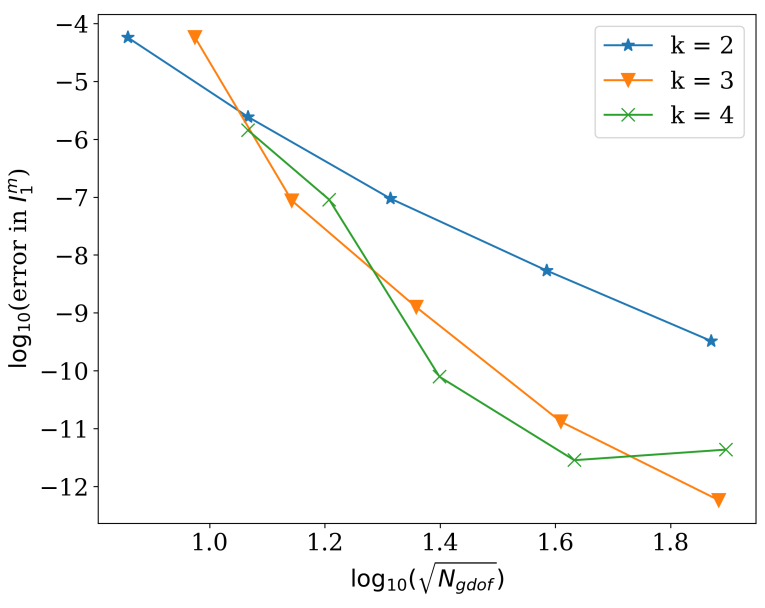

(b) Relative error in $I_{1}^{m}$ from IGA code

Figure 13: The IGA results for the same pincell problem.

The graphs for the IGA thermal energy group neutron scalar fluxes are very similar to the ones shown above so have been omitted for brevity. As can be seen in figures 13a and 13b the IGA solution exhibits better convergence for higher order IGA schemes. This means that for IGA the remaining sources of error after the elimination of geometric error are in the representation of the solution in the basis functions of IGA and are therefore reducible by order elevation. That concludes this section on the effect of geometric error. A more quantitative and rigorous description of the effect geometric error on the VEM solution is beyond the scope of this paper but will be investigated in future work.

It should be noted that typically neutron transport methods are utilised to solve nuclear fuel pincell problems such as the one we have analysed. This is because the physical assumptions underpinning neutron diffusion theory are not typically satisfied for nuclear fuel pincell problems [1]. However the results presented here are compared against another neutron diffusion code so this source of error is not visible. Had the CG-VEM solution been compared to a neutron transport solution, the dominant source of error would have been the discrepancy between the diffusion model and the transport model, not the geometric error. However, the subject of this paper is the application of the VEM to the NDE, not the suitability of the neutron diffusion approximation to particular problems, so further discussion is beyond the scope of this paper.

\subsubsection{Full C5G7 Nuclear Reactor Physics Verification Benchmark Problem}

This section presents the results of the full two-dimensional (2D) C5G7 benchmark. The quantities of interest (QoIs) in this benchmark are as follows:

1. The value of $\mathrm{K}_{\text {eff }}$.

2. The maximum pin power $\mathrm{P}_{\max }$ :

3. The minimum pin power $\mathrm{P}_{\min }$ :

4. The power in the MOX assemblies $\mathrm{P}_{\mathrm{MOX}}$ :

5. The power in the inner $\mathrm{UO} 2$ assembly $\mathrm{P}_{\mathrm{UO} 2}^{i}$.

6. The power in the outer $\mathrm{UO} 2$ assembly. $\mathrm{P}_{\mathrm{UO} 2}^{\mathrm{O}}$. 
The full mathematical definitions of each of these quantities may be found in the appendix. Note that the neutron scalar flux has to be normalised such that:

$$
\int_{V} \sum_{g=1}^{G} \nu \Sigma_{f}^{g}(\mathbf{x}) \phi_{g}(\mathbf{x}) d x=1,060 .
$$

The normalisation of 1,060 is specified in [78] which states that the average pin power must be equal to 1 fission/sec/cell. There are $34^{2}=1,156$ pins in the full assembly minus the 96 guide tubes that do not contribute to the fission source yielding a value of 1,060 . The C5G7 was solved using a series of increasingly more refined spatial meshes containing 10,693, 28,900, 59,245 and 83,232 elements and for orders $k=1,2,3,4$. These meshes were generated by starting with a pincell mesh and tiling to create an array. This pin array was then merged with an L-shaped Cartesian mesh representing the surronding water reflector to produce the quarter core mesh. Some examples of the nuclear fuel pincell meshes are shown in figure 14 . The reference solution to this problem was generated using a CG-VEM scheme of order $k=4$ on the 83,232 element mesh resulting in a discretisation with 1,324,097 degrees of freedom per energy group. The errors in the QoIs presented for the other solutions are with respect to this reference case. To ensure that the reference case itself is correct it was compared to the reference solution from [21], where a high-fidelity IGA neutron diffusion code was used with 3,154,320 degrees of freedom per energy group. The values of both the CG-VEM reference solution and the IGA reference solution are tabulated below in table 4 .
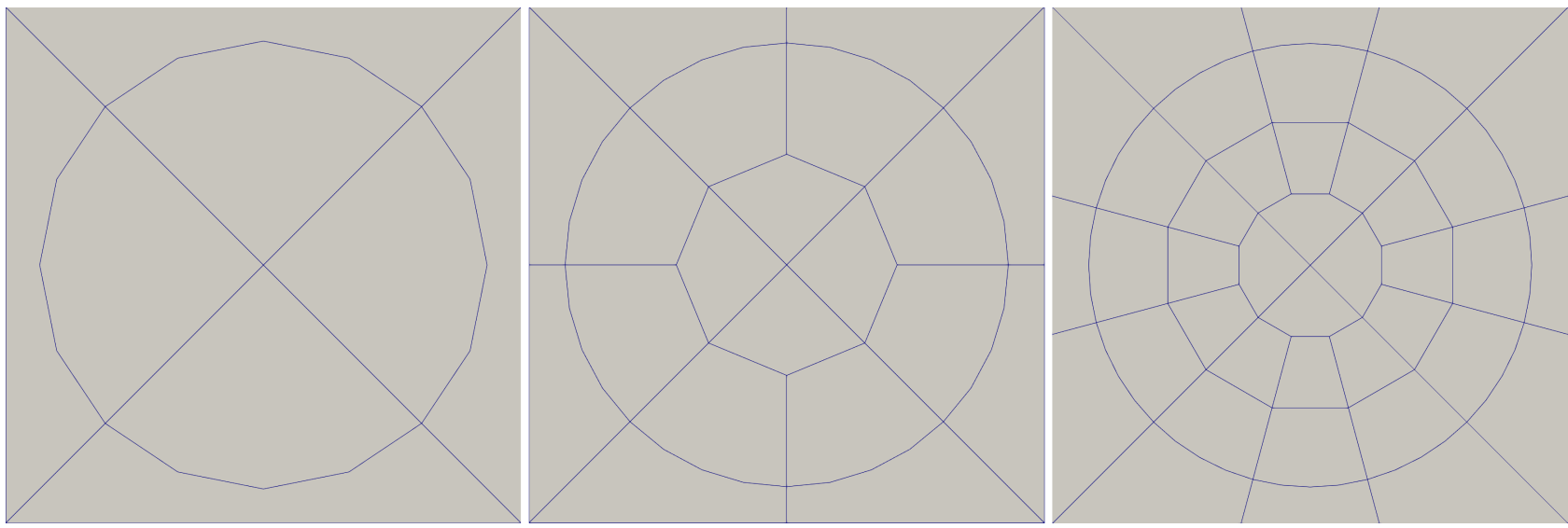

Figure 14: The pincell sub-meshes of the C5G7 meshes containing 10693, 28900 and 59245 elements.

\begin{tabular}{|c|c|c|}
\hline QoI & CG-VEM reference case & IGA case \\
\hline $\mathrm{K}_{\text {eff }}^{\text {ref }}$ & $1.18324079 \mathrm{e}+00$ & $1.1832383 \mathrm{e}+00$ \\
$\mathrm{P}_{\max }$ & $2.53044674 \mathrm{e}+00$ & $2.53044595 \mathrm{e}+00$ \\
$\mathrm{P}_{\min }$ & $2.41548538 \mathrm{e}-01$ & $2.41544689 \mathrm{e}-01$ \\
$\mathrm{P}_{\mathrm{MOX}}$ & $2.11620168 \mathrm{e}+02$ & $2.11620367 \mathrm{e}+02$ \\
$\mathrm{P}_{\mathrm{UO} 2}^{i}$ & $4.97331117 \mathrm{e}+02$ & $4.97230682 \mathrm{e}+02$ \\
$\mathrm{P}_{\mathrm{UO} 2}^{\mathrm{o}}$ & $1.39428546 \mathrm{e}+02$ & $1.39428184 \mathrm{e}+02$ \\
\hline
\end{tabular}

Table 4: The values of the QoIs for the reference case and the IGA case.

As may be seen from table 4 there is very good agreement between the reference CG-VEM case and the IGA case. However, exact convergence of the CG-VEM solution to the IGA is unlikely as the IGA can exactly represent the geometrical domain. Figure 15 presents the errors in 
the QoIs relative to the reference VEM solution in table 4. The power iteration convergence tolerance (equation 3.41) for this problem was set to $1 \times 10^{-13}$.

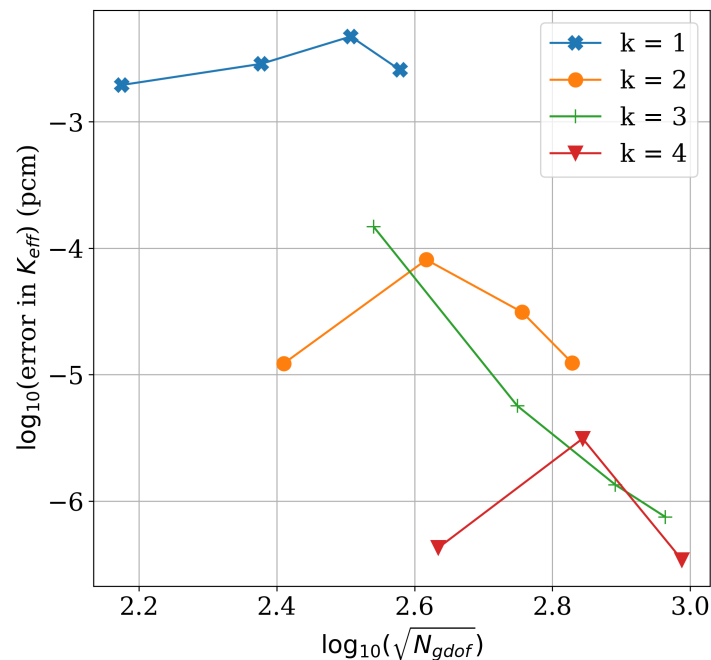

(a) Error in $K_{\text {eff }}$

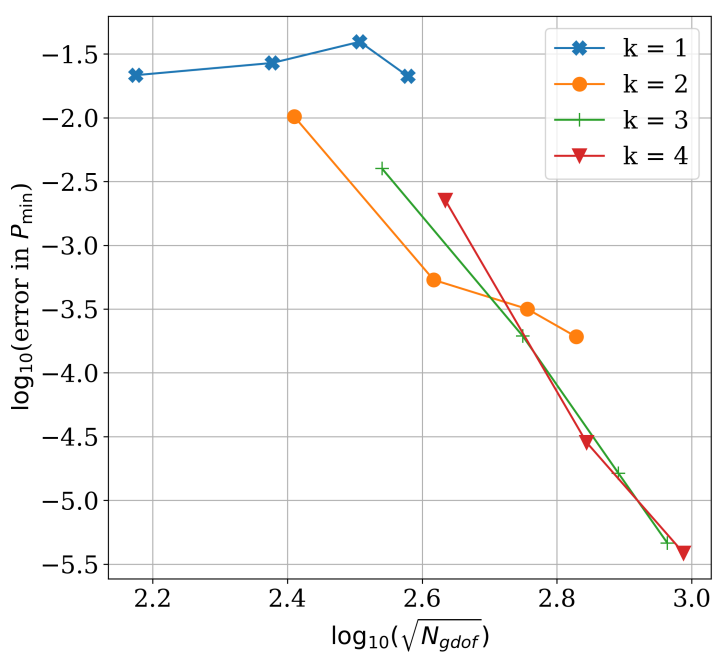

(c) Error in $P_{\max }$

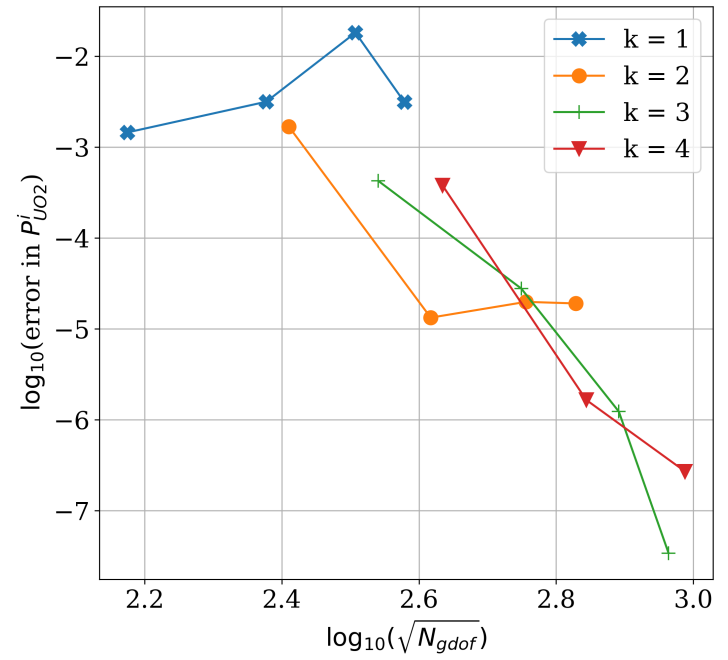

(e) Error in $P_{\mathrm{UO} 2}^{i}$

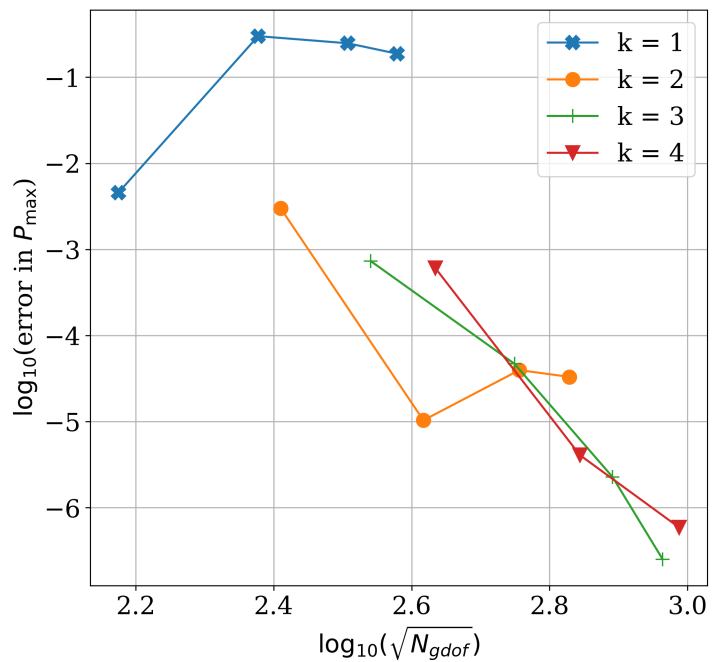

(b) Error in $P_{\max }$

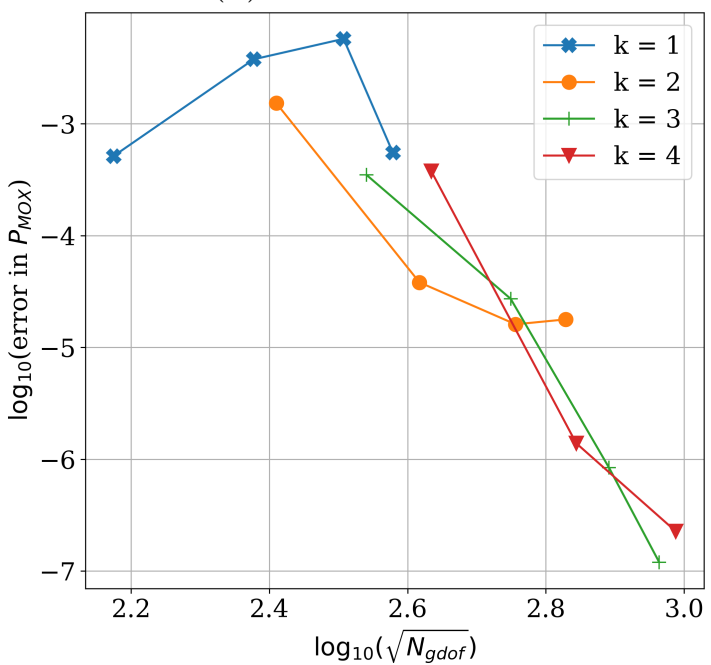

(d) Error in $P_{\text {MOX }}$

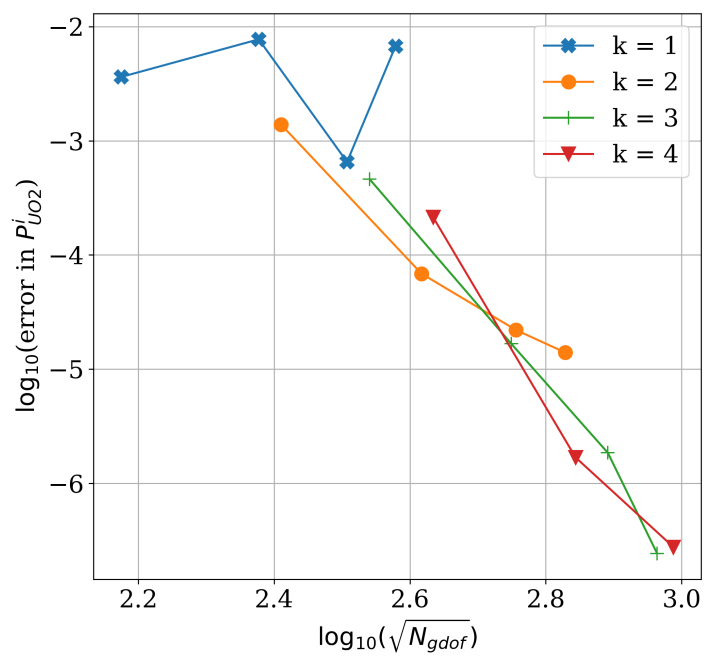

(f) Error in $P_{\mathrm{UO} 2}^{o}$

Figure 15: The C5G7 results, all errors are with respect to the values in the reference case column of table 4

The first point to note about the results in figure 15 is the proximity of the $k=3,4$ curves 
for the integral quantities. This suggests that there is a source of error in those schemes that becomes dominant and which is not improved by order elevation. The most likely source is the geometric error discussed in section 4.2.1. It can be observed that the results from the IAEA benchmark (figures 6 and 7) do not exhibit this property. In the IAEA benchmark, which does not contain any curved edges, the higher order convergence curves are shifted downward relative to the $k=2$ curve. Therefore, the dominant sources of error are from the representation of the solution in the VEM basis and consequently may be reduced by order elevation. With that said the CG-VEM still attains very low errors in C5G7 benchmark albeit at a lower order of convergence for the $k=3,4$ schemes. An important point to note about the C5G7 mesh is the small number of elements per pincell compared to a FE triangular mesh (such as those presented in appendix B of [78]). Something made possible by the fact that the CG-VEM may use general n-sided polygons. This fact was exploited to introduce extra edges on the interface between the fuel and the moderator and thus reduce the geometric error for a given number of elements per pincell. Such mesh manipulations are possible in VEM and provide new possibilities for mesh generation in nuclear reactor physics. In addition to the errors in the QoIs presented in figure 15 some neutron scalar flux contours are also presented below in figure 16, these were the neutron scalar flux contours generated by the 83,232 element mesh at VEM order $k=4$.

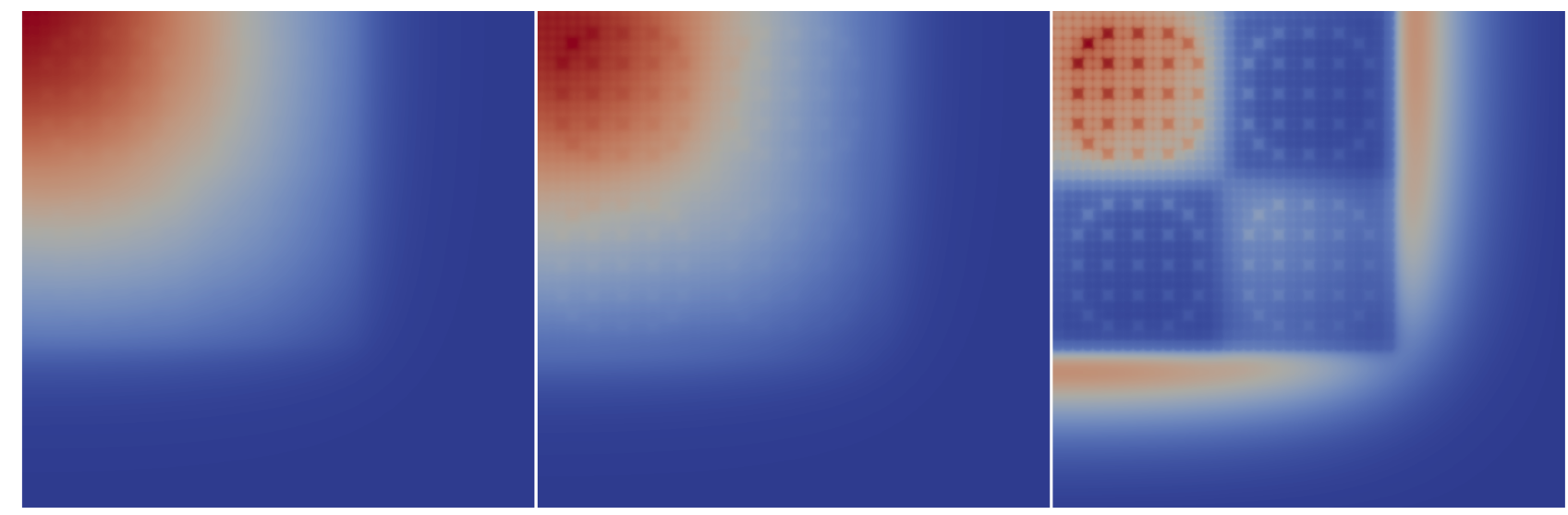

Figure 16: The C5G7 neutron scalar flux contours for the group $g=1$ (left), $g=4$ (middle) and $g=7$ (right) fluxes.

As may be seen from figure 16 the energy dependent neutron scalar fluxes behave physically as one would expect. The highest neutron energy group, $g=1$, is called the fast group. From the contour plot in figure 16 this shows that fast neutrons are produced during prompt neutron fission in the fuel assemblies as one would expect. Therefore, because of the quarter symmetry of the C5G7 benchmark the fast neutron scalar flux is highest in the central UOX fuel assembly. The epi-thermal energy group, $g=4$, covers the resonance energy region so this again reaches a maximum in the central UOX fuel assembly with a much lower epi-thermal neutron scalar flux within the adjacent MOX fuel assemblies due to significant absorption of the epi-thermal neutrons. The thermal energy group, $g=7$, has a relatively high neutron scalar flux within the central UOX fuel assembly and the reflector region surrounding the fuel assemblies. The relatively high thermal neutron scalar flux in the central UOX fuel assembly is due to neutron slowing down and thermalisation within the moderator region surrounding each nuclear fuel pin. Likewise, the relatively high thermal neutron scalar flux within the reflector region is due to neutrons slowing down and thermalising. That concludes the numerical results section of this paper. 


\section{Conclusion}

This paper presents a number of key numerical results associated with the VEM method. First, the paper presented the first application of the CG-VEM to the field of nuclear reactor physics, specifically to the multi-group neutron diffusion equation and demonstrated the versatility of the mesh topology admissible in the VEM for the purposes of solving the NDE. This was accomplished by solving the two-dimensional (2D) IAEA and C5G7 nuclear reactor physics verification benchmark problems with both Cartesian and polygonal meshes. Second, it replicated the convergence rates presented in $[4,49]$ for various types of VEM meshes using a standard MMS procedure. Third, it demonstrated the theoretical convergence rates of the CGVEM for problems with continuous solutions but discontinuous coefficients and discontinuous source functions, a situation that often arises in neutron diffusion problems.

Mathematically solving the NDE requires the solution of an arbitrary number of coupled elliptic PDEs with discontinuous material properties and sources from neutron fission and scattering. While the underlying CG-VEM discretisation presented in this paper is not original the problem it was applied to is more complicated in terms of geometry and material properties than has been presented in previous work. Most of the VEM research literature, currently published, typically solve relatively simplistic problems that are specifically selected to demonstrate the numerical properties of the discretisation scheme. The versatility of the mesh topology admissible in VEM ameliorates and overcomes many of the major challenges posed by multi-dimensional mesh generation [61, 62, 63, 64, 65]; as well as simplifying adaptive mesh refinement (AMR). For instance in non-conforming AMR hanging nodes are generated and these are incorporated naturally within the VEM spatial discretisation method as extra vertices.

The key drawback of the VEM, as presented in this paper, is that it cannot represent geometries with curved boundaries exactly. Therefore, it cannot exactly represent the geometry of nuclear reactor designs that contain circular nuclear fuel pins. This obviates the benefits of order elevation in cases where the problem geometry contains curves and subsequently reduces the advantage of VEM over current FE based spatial discretisation methods. Therefore, the application of the exact-geometry VEM presented in [79] is an important priority in future research in this field. In this paper the VEM meshes used to represent the neutron scalar flux of each neutron energy group were identical. Future improvements on the present work would include AMR with mesh-to-mesh interpolation algorithms for polygonal and polyhedral meshes, such as those presented in [80, 81, 82], with different spatial refinements for each different neutron energy group. This will be very important if AMR and energy-dependant spatial mesh algorithms are to be fully developed and exploited as they are for IGA [22, 72]. 


\section{Appendix}

\subsection{Method of Manufactured solutions (MMS)}

The error measure used in these MMS tests is the $L^{2}(V)$ measure defined as [39]:

$$
e_{L^{2}}=\sqrt{\sum_{E \in \mathcal{T}_{h}}\left\|\phi_{\mathrm{mms}}(\mathbf{x})-\Pi_{k}^{0} \phi_{h}(\mathbf{x})\right\|_{0, E}^{2}},
$$

where $\phi_{\mathrm{mms}}(\mathbf{x})$ is the chosen analytic solution to the PDE problem under consideration. In the following two MMS test cases let $\mathcal{T}_{h}$ denote the mesh of domain $V \subset \mathbb{R}^{2}$ and let $\mathcal{E}_{h}$ denote the set of edges in $\mathcal{T}_{h}$ that are coincident with the boundary $\partial V$.

\subsubsection{MMS Verification Test Case 1}

This MMS verification test case was developed to demonstrate the robustness of the CG-VEM convergence rate to mesh distortion and element concavity. In this test case the domain was chosen to be the unit square $V=[0,1]^{2}$. The PDE under consideration is the linear reactiondiffusion equation with unit diffusion and reaction coefficients:

$$
\left\{\begin{array}{l}
-\nabla^{2} \phi(\mathbf{x})+\phi(\mathbf{x})=f(\mathbf{x}) \quad \forall \mathbf{x} \in V . \\
-\nabla \phi(\mathbf{x}) \cdot \mathbf{n}+\phi(\mathbf{x})=r(\mathbf{x}) \quad \forall \mathbf{x} \in \partial V
\end{array}\right.
$$

Both $f(\mathbf{x})$ and $r(\mathbf{x})$ were prescribed such that the chosen analytic solution would be:

$$
\phi_{\mathrm{mms}}=(x+\cos (2 \pi y))(y-\sin (2 \pi x)) .
$$

The discrete variational problem for equation 6.2 is:

$$
\left\{\begin{array}{l}
\text { Find } \phi_{h} \in \mathcal{V}_{k}(V) \text { such that: } \\
A_{h}\left(\phi_{h}, v_{h}\right)=l_{h}\left(v_{h}\right) \quad \forall v_{h} \in \mathcal{V}_{k}(V)
\end{array}\right.
$$

where:

$$
\begin{gathered}
A_{h}\left(\phi_{h}, v_{h}\right)=\sum_{E \in \mathcal{T}_{h}} a_{h}^{E}\left(\phi_{h}, v_{h}\right)+b_{h}^{E}\left(\phi_{h}, v_{h}\right)+\sum_{e \in \mathcal{E}_{h}}\left(\phi_{h}, v_{h}\right)_{0, e} \\
l_{h}\left(v_{h}\right)=\sum_{E \in \mathcal{T}_{h}} f_{h}^{E}\left(v_{h}\right)+\sum_{e \in \mathcal{E}_{h}}\left(r, v_{h}\right)_{0, e}
\end{gathered}
$$

The terms $a_{h}^{E}\left(\phi_{h}, v_{h}\right), b_{h}^{E}\left(\left(\phi_{h}, v_{h}\right)\right)$ and $f_{h}^{E}\left(v_{h}\right)$ were introduced in section 2.3, equations 2.24, 2.25 and 2.26 respectively. If $f(\mathbf{x}) \in H^{s-2}(V)$ and $r(\mathbf{x}) \in H^{s-3 / 2}(\partial V)$ then $u_{h} \in H^{s}(V)$ (see [83], chapter 3.1.3). Under these assumptions on the data the CG-VEM solution to equation 6.4 satisfies the following error estimate [39]:

$$
\left\|\phi_{\mathrm{mms}}(\mathbf{x})-\phi_{h}(\mathbf{x})\right\|_{0, \mathcal{T}_{h}} \leq C h^{s}\left(|u|_{s, \mathcal{T}_{h}}+|f|_{s-2, \mathcal{T}_{h}}+|r|_{s-3 / 2, \mathcal{E}_{h}}\right) .
$$


In this case $f(\mathbf{x})$ and $r(\mathbf{x})$ are derived from $\phi_{\mathrm{mms}}(\mathbf{x})$, which is infinitely differentiable, therefore $s=k+1$. Equation 6.4 was solved on four different types of mesh shown in figure 17 .
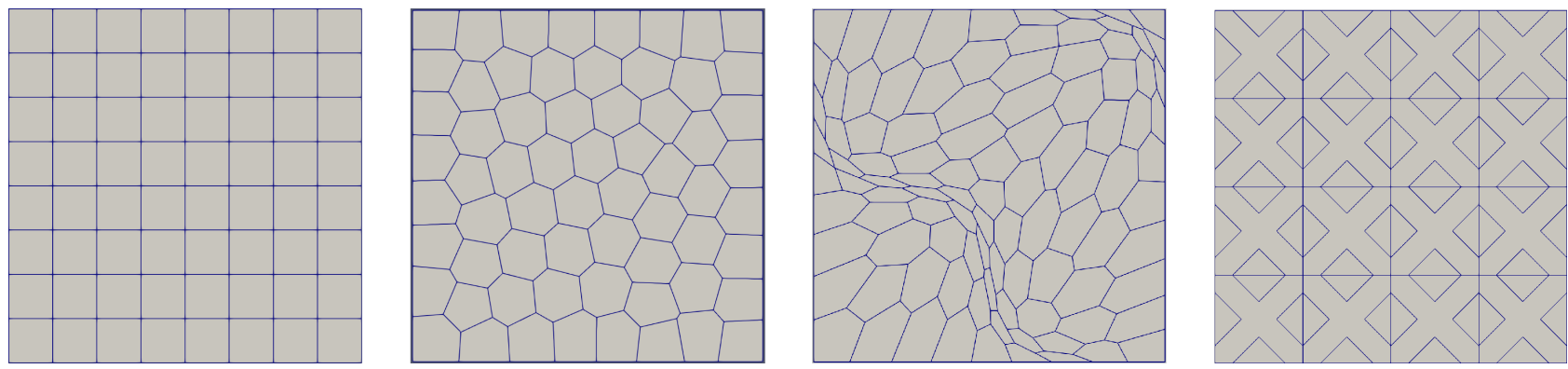

Figure 17: The four different type of mesh used in the MMS tests. They are labelled $\mathcal{T}_{h}^{1}, \mathcal{T}_{h}^{2}, \mathcal{T}_{h}^{3}$ and $\mathcal{T}_{h}^{4}$ from left to right.

Mesh $\mathcal{T}_{h}^{1}$ is a standard Cartesian mesh, $\mathcal{T}_{h}^{2}$ was generated by means of a centroidal Voronoi tessellation (CVT) and $\mathcal{T}_{h}^{3}$ was simply $\mathcal{T}_{h}^{2}$ with the vertex coordinates altered with the following transform taken from [34]:

$$
\hat{x}=x+0.12(\sin (2 \pi x) \sin (2 \pi y)) \quad \hat{y}=y+0.12(\sin (2 \pi x) \sin (2 \pi y)) .
$$

In mesh $\mathcal{T}_{h}^{4}$ each square is comprised of four triangles and a central cross that is a single element. The convergence curves for this test case are shown in the appendix in figure 18 and the observed convergence rates with respect to element diameter $h$ are tabulated in table 5:

\begin{tabular}{c|c|c|c|c} 
VEM order $k$ & Cartesian $\left(\mathcal{T}_{h}^{1}\right)$ & CVT $\left(\mathcal{T}_{h}^{2}\right)$ & Dist-CVT $\left(\mathcal{T}_{h}^{3}\right)$ & Cross $\left(\mathcal{T}_{h}^{4}\right)$ \\
\hline 1 & 1.99 & 2.02 & 1.88 & 1.62 \\
2 & 2.98 & 3.03 & 2.94 & 2.96 \\
3 & 4.01 & 4.16 & 4.03 & 3.98 \\
4 & 5.00 & 4.95 & 4.77 & 5.02
\end{tabular}

Table 5: Table of observed convergence rates, each one corresponds to the slope of one of the convergence curves in figures $18 \mathrm{a}$ to $18 \mathrm{~d}$.

The tabulated values are in good agreement the error estimate of $k+1$ for a VEM scheme of order $k$ in the $L_{2}$ norm. The convergence curves for this MMS test case are shown in figure 18: 


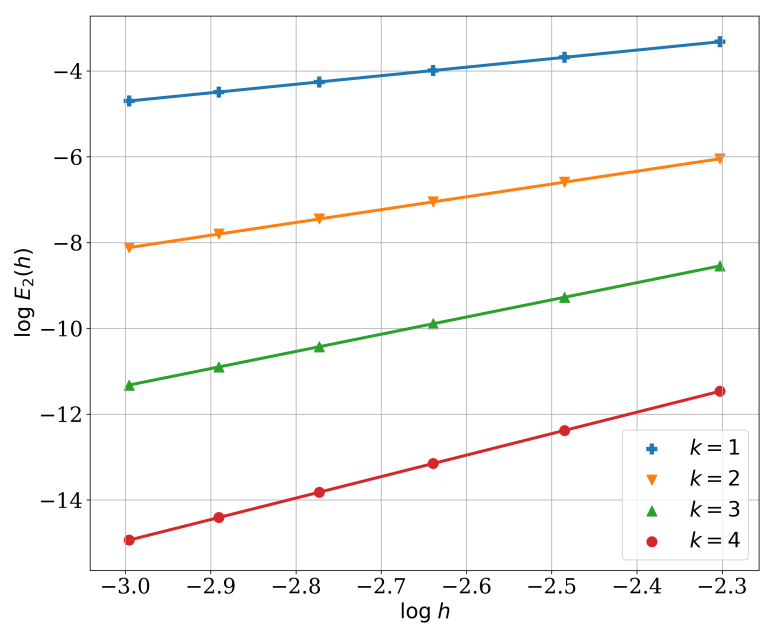

(a) Cartesian mesh $\mathcal{T}_{h}^{1}$ convergence plots.

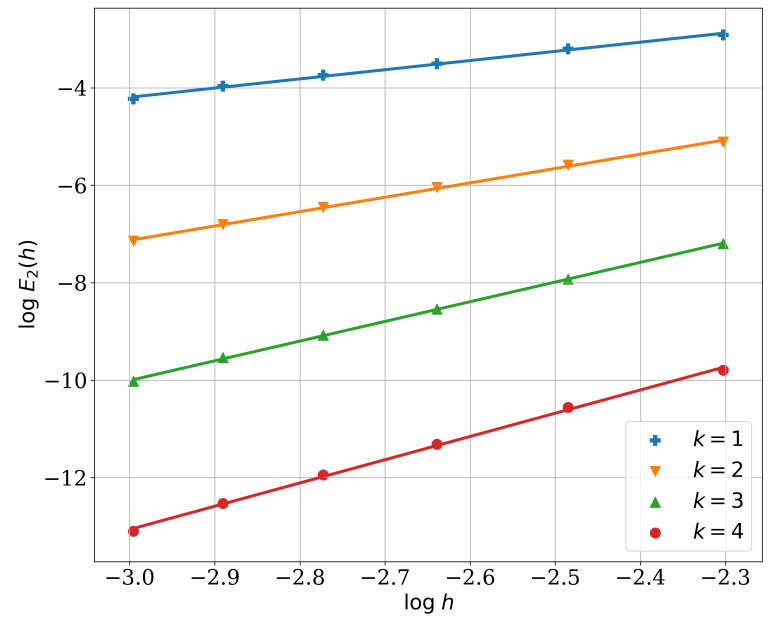

(c) Distorted CVT mesh $\mathcal{T}_{h}^{3}$ convergence plots.

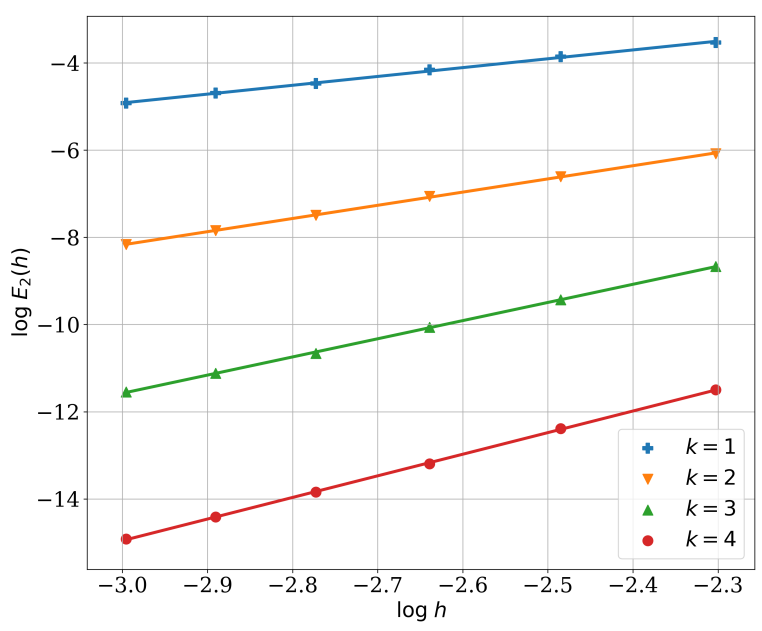

(b) CVT mesh $\mathcal{T}_{h}^{2}$ convergence plots.

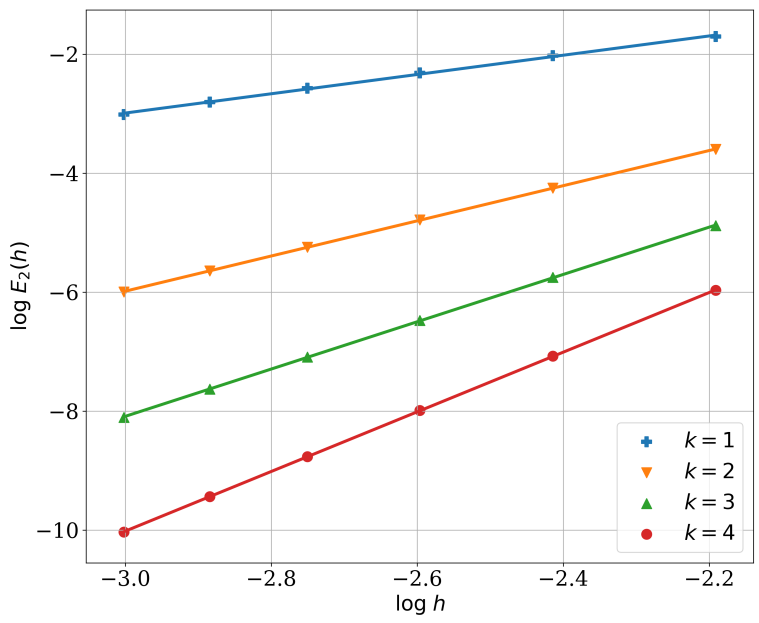

(d) Cross mesh $\mathcal{T}_{h}^{4}$ convergence plots.

Figure 18: The results of the first MMS verification test case.

\subsubsection{MMS Verification Test Case 2}

This MMS verification test case was undertaken to simulate more closely the sort of problems encountered in neutron diffusion simulations, namely problems involving piecewise constant material coefficients and piecewise smooth source functions. The domain was the unit square $V=[0,1]^{2}$ with the bottom and top halves possessing different diffusion and reaction coefficients. The sequence of meshes employed in this test were simply Cartesian meshes that conform to the internal interface i.e. no element in the mesh crosses the boundary between $V_{1}$ and $V_{2}$. This is in keeping with the kinds of meshes employed to model nuclear reactor cores where the mesh must also conform to the interfaces between materials.

The equations to solve are:

$$
\left\{\begin{array}{l}
\nabla \cdot(-D(\mathbf{x}) \nabla \phi(\mathbf{x}))+\Sigma(\mathbf{x}) \phi(\mathbf{x})=f(\mathbf{x}) \quad \forall \mathbf{x} \in V \\
(-D(\mathbf{x}) \nabla \phi(\mathbf{x})) \cdot \mathbf{n}+\phi(\mathbf{x})=r(\mathbf{x}) \quad \forall \mathbf{x} \in \partial V
\end{array}\right.
$$

The discrete variational formulation of equation is almost identical to equation 6.4 with the only difference being the stiffness and mass bilinear forms are scaled by the diffusion and reaction coefficients. Given that $D(\mathbf{x}), \Sigma(\mathbf{x}) \in L^{\infty} V$ the error estimate of 6.7 still holds. Let $\mathbf{x}=(x, y)^{T}$, 


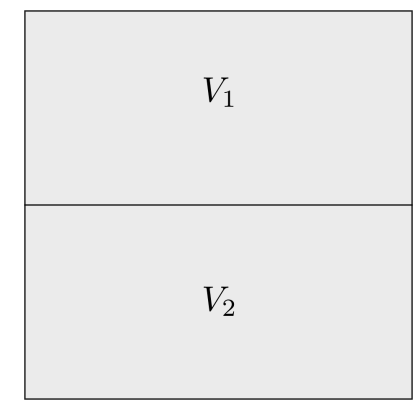

Figure 19: Computational domain for the second MMS test case where $V=V_{1} \cup V_{2}$.

then $D(\mathbf{x})$ and $\Sigma(\mathbf{x})$ are given by:

$$
D(\mathbf{x})=D_{2}+\left(D_{1}-D_{2}\right) H(y-0.5) \quad \text { and } \quad \Sigma(\mathbf{x})=\Sigma_{2}+\left(\Sigma_{1}-\Sigma_{2}\right) H(y-0.5),
$$

where $H(x)$ is the Heaviside step function. The MMS solution $\phi_{m m s}$ was chosen to be the same as the previous MMS test, equation 6.3. Therefore, $\phi_{m m s}(\mathbf{x})$ is a smooth function almost everywhere (it is singular at the interface between $V_{1}$ and $V_{2}$ ) meaning the MMS source function $f(\mathbf{x})$ is only piecewise continuous with a discontinuity at the internal boundary between $V_{1}$ and $V_{2}$. By using the chain rule on the diffusion term $f(\mathbf{x})$ is computed as follows:

$$
\begin{aligned}
f(\mathbf{x}) & =-\nabla D(\mathbf{x}) \cdot \nabla \phi_{m m s}(\mathbf{x})-D(\mathbf{x}) \nabla^{2} \phi_{m m s}(\mathbf{x})+\Sigma(\mathbf{x}) \phi_{m m s}(\mathbf{x}) \\
& =-\frac{\partial D(\mathbf{x})}{\partial y} \frac{\partial \phi_{m m s}(\mathbf{x})}{\partial y}-D(\mathbf{x}) \nabla^{2} \phi_{m m s}(\mathbf{x})+\Sigma(\mathbf{x}) \phi_{m m s}(\mathbf{x}) \\
& =-\left(D_{1}-D_{2}\right) \delta(y-0.5) \frac{\partial \phi_{m m s}(\mathbf{x})}{\partial y}-D(\mathbf{x}) \nabla^{2} \phi_{m m s}(\mathbf{x})+\Sigma(\mathbf{x}) \phi_{m m s}(\mathbf{x}),
\end{aligned}
$$

where $\delta(y-0.5)$ is the Dirac delta distribution. The first term of $f(\mathbf{x})$ is a line source that runs along the internal boundary $V_{1} \cap V_{2}$. For the purposes of computing the global source vector the MMS source function is split into the piecewise continuous part $f_{1}$ and the line source $f_{2}$ :

$$
f_{1}(\mathbf{x})=-D(\mathbf{x}) \nabla^{2} \phi_{m m s}(\mathbf{x})+\Sigma(\mathbf{x}) \phi_{m m s}(\mathbf{x}) \quad f_{2}(\mathbf{x})=-\left(D_{1}-D_{2}\right) \delta(y-0.5) \frac{\partial \phi_{m m s}(\mathbf{x})}{\partial y}
$$

Letting $\mathcal{E}_{h}^{*}$ denote the set of edges in the mesh $\mathcal{T}_{h}$ that lie on $V_{1} \cap V_{2}$ then global source vector may be written as:

$$
\mathbf{f}=\sum_{E \in \mathcal{T}_{h}} \mathbf{f}_{E}^{1}+\sum_{e \in \mathcal{E}_{h}^{*}} \mathbf{f}_{e}^{2}
$$

where:

$$
\left(\mathbf{f}_{E}^{1}\right)_{i}=\int_{E} f_{1}(\mathbf{x}) \Pi_{k}^{0} \varphi_{i}(\mathbf{x}) d x \quad\left(\mathbf{f}_{e}^{2}\right)_{i}=-\left(D_{1}-D_{2}\right) \int_{e} \frac{\partial \phi_{m m s}(\mathbf{x})}{\partial y} \varphi_{i} d x
$$

The local source vectors $\mathbf{f}_{E}^{1}$ are the standard VEM source vectors introduced in section 2 while 
the local source vectors on edges $\mathbf{f}_{e}^{1}$ are simply the source vectors of 1D Gauss-Lobatto spectral elements, a description of which may be found in chapter 3 of [84]. Figure 20 illustrates the one-dimensional (1D) Lobatto spectral elements on the $V_{1} \cap V_{2}$ interface.
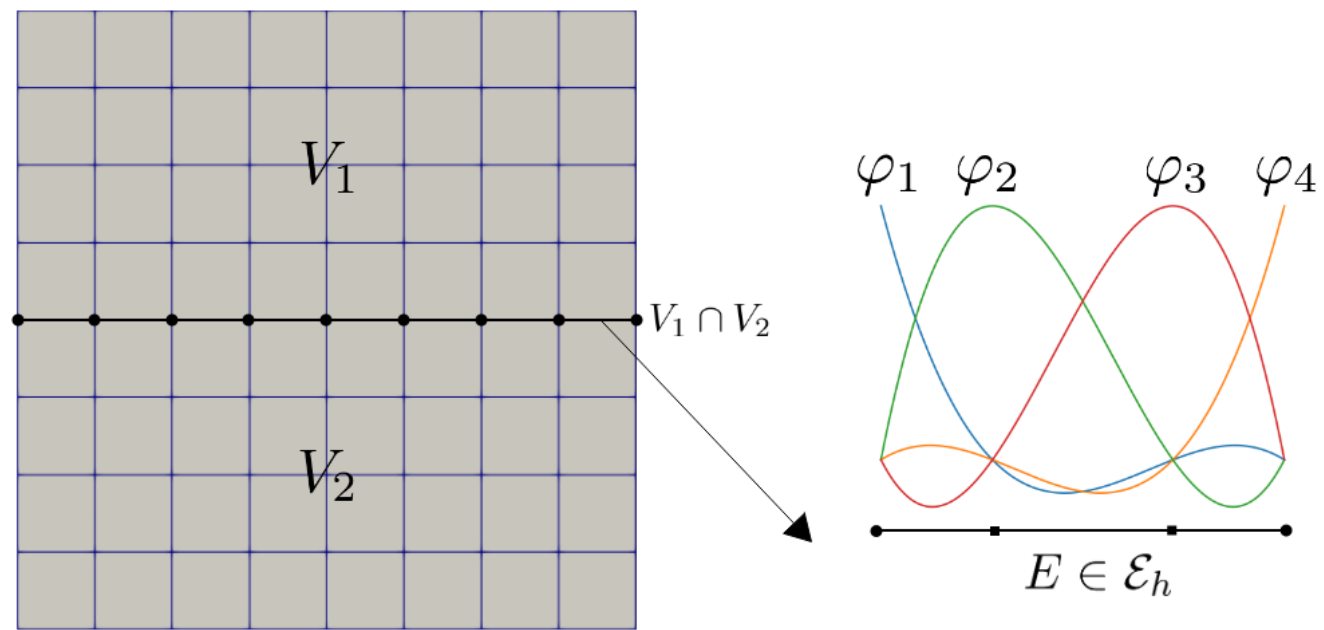

Figure 20: An illustration of the mesh $\mathcal{T}_{h}$ with edge set $\mathcal{E}_{h}^{*}$ shown in black and an example of a 1D spectral element for $k=3$.

Four material configurations were tested and they are tabulated in table $6:$

\begin{tabular}{c|c|c|c|c} 
Configuration & $D_{1}$ & $\Sigma_{1}$ & $D_{2}$ & $\Sigma_{2}$ \\
\hline 1 & 1 & 1 & 10 & 1 \\
2 & 1 & 1 & 100 & 1 \\
3 & 1 & 1 & 1 & 10 \\
4 & 1 & 1 & 1 & 100
\end{tabular}

Table 6: Table of coefficients for each configuration.

Configurations 1 and 2 prescribe the reaction coefficients equal and vary the diffusion coefficients while configurations 3 and 4 do the opposite. For each of these configurations equation 6.1.2 was solved for orders $k=1,2,3,4$ on Cartesian meshes with 100, 144, 196, 256 and 324 elements each. The convergence plots for the above cases are provided in the appendix in figure 21 . The observed convergence rates are tabulated below in table 7 :

\begin{tabular}{c|c|c|c|c}
\multirow{2}{*}{ VEM order $k$} & \multicolumn{4}{|c}{ Configuration } \\
& 1 & 2 & 3 & 4 \\
\hline 1 & 1.97 & 1.97 & 1.97 & 2.01 \\
2 & 3.00 & 3.00 & 3.00 & 2.99 \\
3 & 4.04 & 4.04 & 4.04 & 4.05 \\
4 & 4.96 & 4.96 & 4.96 & 4.97
\end{tabular}

Table 7: Table of observed convergence rates from each of the configurations presented in table 6 .

Additionally, the convergence curves for MMS test in section 6.1.2 are shown in figure 21:

Table 7 shows that the CG-VEM exhibits no degradation in the order of convergence in the presence of diffusion/reaction coefficient heterogeneity and are in agreement with the theoretical 


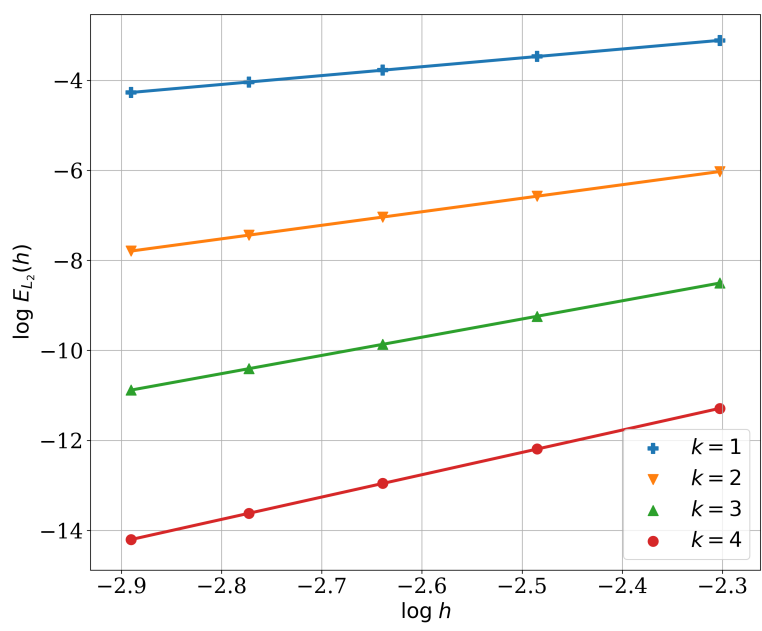

(a) The convergence curves for configuration 1.

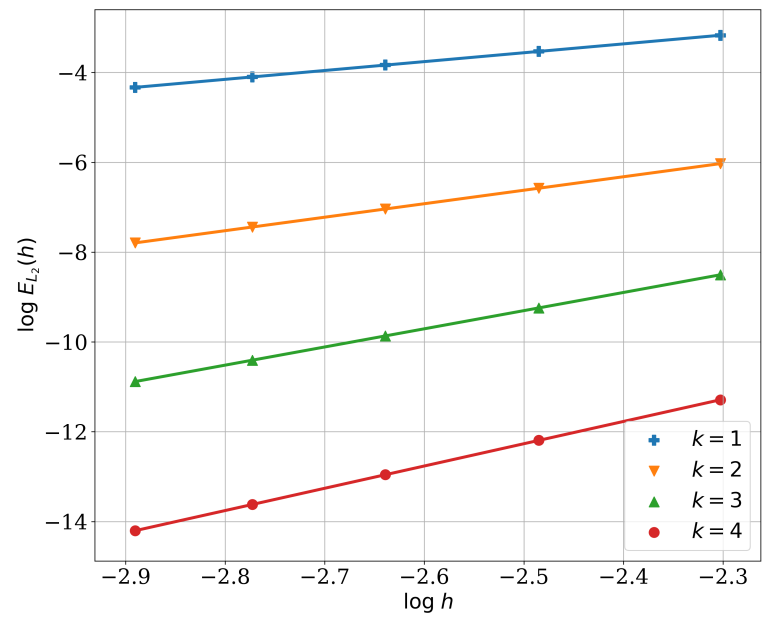

(c) The convergence curves for configuration 3 .

Figure 21: The results of the second MMS verification test case.

estimate of $k+1$. It is important to note that this is only true in the case where the meshes employed conform with the material interface. If non-conforming mesh were used the rate of convergence would be lower. However, the consideration of such cases is beyond the scope of this paper.

\subsection{C5G7 Quantities of interest}

The mathematical definitions of each of the QoIs from section 4.2.2 are: The maximum pin power $\mathrm{P}_{\max }$ :

$$
\mathrm{P}_{\max }=\max _{i} \int_{V_{i}} \sum_{g=1}^{G} \nu \Sigma_{f}^{g}(\mathbf{x}) \phi_{g}(\mathbf{x}) d x
$$

Where $V_{i}$ is the set of points in pin $i$. The minimum pin power $\mathrm{P}_{\min }$ :

$$
\mathrm{P}_{\min }=\min _{i} \int_{V_{i}} \sum_{g=1}^{G} \nu \Sigma_{f}^{g}(\mathbf{x}) \phi_{g}(\mathbf{x}) d x
$$


The power in the MOX assemblies $\mathrm{P}_{\text {MOX: }}$

$$
\mathrm{P}_{\mathrm{MOX}}=\int_{V_{\mathrm{MOX}}} \sum_{g=1}^{G} \nu \Sigma_{f}^{g}(\mathbf{x}) \phi_{g}(\mathbf{x}) d x
$$

Where $V_{\text {MOX }}$ is either of the MOX assemblies. Because of symmetry of the flux field about the line running from $(0,64.26)$ to $(64.26,0)$ the power of both MOX assemblies are equal so only one need be presented. The power in the inner UO2 assembly $\mathrm{P}_{\mathrm{UO} 2}^{i}$.

$$
\mathrm{P}_{\mathrm{UO} 2}^{i}=\int_{V_{\mathrm{U} O 2}^{i}} \sum_{g=1}^{G} \nu \Sigma_{f}^{g}(\mathbf{x}) \phi_{g}(\mathbf{x}) d x
$$

Where $V_{\mathrm{UO} 2}^{i}$ is the inner UO2 assembly. The power in the outer UO2 assembly. $\mathrm{P}_{\mathrm{UO} 2}^{\mathrm{o}}$.

$$
\mathrm{P}_{\mathrm{UO} 2}^{\mathrm{o}}=\int_{V_{\mathrm{U} 2}^{\mathrm{o}} 2} \sum_{g=1}^{G} \nu \Sigma_{f}^{g}(\mathbf{x}) \phi_{g}(\mathbf{x}) d x
$$

Where $V_{\mathrm{UO} 2}^{\mathrm{o}}$ is the outer UO2 assembly.

\section{Acknowledgements}

Mr J. Ferguson would like to acknowledge the Engineering and Physical Sciences Research Council (EPSRC) through the Doctoral Training Award (DTA) PhD scheme (EPSRC Grant No.: EP/R512540/1). Mr J. Ferguson also acknowledges the industrial support of Rolls-Royce. Mr J. Ferguson would also like to thank Dr C. Latimer for providing isogeometric reference results for the C5G7 UO2 Nuclear Fuel Pincell Problem and the 2D IAEA Nuclear Reactor Physics Verification Benchmark Problem. Dr M.D. Eaton and Dr J. Kópházi would like to thank the Engineering and Physical Sciences Reasearch Council (EPSRC) for their support through the following grants: Adaptive Hierarchical Radiation Transport Methods to Meet Future Challenges in Reactor Physics (EPSRC Grant No.: EP/ J002011/1) and RADIANT: A Parallel, Scalable, High Performance Radiation Transport Modelling and Simulation Framework for Reactor Physics, Nuclear Criticality Safety Assessment and Radiation Shielding Analyses (EPSRC Grant No.: EP/K503733/1). The authors would like to thank Mr M. Harvey, who is the research computing services (RCS) manager, and his team at Imperial College London. Finally, the authors would like to thank the anonymous reviewers for their suggestions.

\section{Data Statement}

In accordance with EPSRC funding requirements all supporting data used to create figures in this paper may be accessed at the following URL: https://doi.org/10.5281/zenodo.4036048 


\section{References}

[1] E. E. Lewis, W. F. Miller Jr, Computational Methods of Neutron Transport, American Nuclear Society (ANS), La Grange Park, Illinois, USA, 1993.

URL http://www.ans.org/store/item-350016/

[2] L. S. Ornstein, G. E. Uhlenbeck, Some kinetic problems regarding the motion of neutrons through paraffine, Physica 4 (6) (1937) 478-486. doi:10.1016/s0031-8914(37)80080-4.

[3] A. Hèbert, Applied Reactor Physics, 2nd Edition, Presses Internationales Polytechnique, 2016.

[4] L. B. da Veiga, F. Brezzi, A. Cangiani, G. Manzizni, L. D. Marini, A. Russo, Basic principles of virtual element methods, Mathematical Models and Methods in Applied Sciences 23 (01) (2013) 199-214. doi:10.1142/s0218202512500492.

[5] Y. A. Enrico Sartori, Nuclear Computational Science, Springer-Verlag GmbH, 2010. doi: 10.1007/978-90-481-3411-3.

[6] R. Sanchez, Assembly homogenization techniques for core calculations, Progress in Nuclear Energy 51 (1) (2009) 14-31. doi:10.1016/j.pnucene.2008.01.009.

[7] K. S. Smith, Assembly homogenization techniques for light water reactor analysis, Progress in Nuclear Energy 17 (3) (1986) 303-335. doi:10.1016/0149-1970 (86)90035-1.

[8] R. D. Lawrence, Progress in nodal methods for the solution of the neutron diffusion and transport equations, Progress in Nuclear Energy 17 (3) (1986) 271-301. doi:10.1016/ 0149-1970 (86) 90034-x.

[9] M. D. Brough, C. T. Chudley, Characteristic ray solutions of the transport equation, in: Advances in Nuclear Science and Technology, Vol. 12, Springer US, 1980, pp. 1-31. doi:10.1007/978-1-4613-9916-2_1.

[10] L. A. Semenza, E. E. Lewis, E. C. Rossow, The application of the finite element method to the multigroup neutron diffusion equation, Nuclear Science and Engineering 47 (3) (1972) 302-310. doi:10.13182/nse72-a22416.

[11] C. M. Kang, K. F. Hansen, Finite element methods for reactor analysis, Nuclear Science and Engineering 51 (4) (1973) 456-495. doi:10.13182/nse73-a23278.

[12] Y. Wang, J. Ragusa, Application of hp adaptivity to the multigroup diffusion equations, Nuclear Science and Engineering 161 (1) (2009) 22-48. doi:10.13182/nse161-22.

[13] Y. Wang, W. Bangerth, J. Ragusa, Three-dimensional h-adaptivity for the multigroup neutron diffusion equations, Progress in Nuclear Energy 51 (3) (2009) 543-555. doi: 10.1016/j.pnucene.2008.11.005.

[14] D. Braess, Finite Elements, Cambridge University Press, Cambridge, UK, 2007. URL https://www. cambridge.org/gb/academic/subjects/mathematics/ numerical-analysis/finite-elements-theory-fast-solvers-and-applications-solid-me format $=\mathrm{PB}$

[15] D. Vartziotis, J. Wipper, M. Papadrakakis, Improving mesh quality and finite element solution accuracy by GETMe smoothing in solving the Poisson equation, Finite Elements in Analysis and Design 66 (2013) 36-52. doi:10.1016/j.finel.2012.11.004. URL https://linkinghub.elsevier.com/retrieve/pii/S0168874X12002077 
[16] P. Frey, Mesh Generation, ISTE Ltd., 2008. doi:10.1002/9780470611166.

[17] J. A. Cottrell, Y. Bazilevs, T. J. R. Hughes, Isogeometric Analysis, John Wiley \& Sons Ltd, Chichester, UK, 2009.

URL https://www.wiley.com/en-gb/Isogeometric+Analysis\%3A+Toward+ Integration+of+CAD+and+FEA-p-9780470749098

[18] A. R. Owens, J. A. Welch, J. Kópházi, M. D. Eaton, Discontinuous isogeometric analysis methods for the first-order form of the neutron transport equation with discrete ordinate $\left(\mathrm{S}_{\mathrm{N}}\right)$ angular discretisation, Journal of Computational Physics 315 (2016) 501-535. doi: $10.1016 / j \cdot j c p .2016 .03 .060$.

[19] S. K. Hall, M. D. Eaton, M. M. R. Williams, The application of isogeometric analysis to the neutron diffusion equation for a pincell problem with an analytic benchmark, Annals of Nuclear Energy 49 (2012) 160-169. doi:10.1016/j.anucene.2012.05.030.

[20] A. R. Owens, J. A. Welch, J. Kópházi, M. D. Eaton, An adaptive, hanging-node, discontinuous isogeometric analysis method for the first-order form of the neutron transport equation with discrete ordinate $\left(\mathrm{S}_{\mathrm{N}}\right)$ angular discretisation, Computer Methods in Applied Mechanics and Engineering 318 (2017) 215-241. doi:10.1016/j.cma.2017.01.036.

[21] J. A. Welch, J. Kópházi, A. R. Owens, M. D. Eaton, Isogeometric analysis for the multigroup neutron diffusion equation with applications in reactor physics, Annals of Nuclear Energy 101 (2017) 465-480. doi:10.1016/j.anucene.2016.11.015.

[22] J. A. Welch, J. Kópházi, A. R. Owens, M. D. Eaton, A geometry preserving, conservative, mesh-to-mesh isogeometric interpolation algorithm for spatial adaptivity of the multigroup, second-order even-parity form of the neutron transport equation, Journal of Computational Physics 347 (2017) 129-146. doi:10.1016/j.jcp.2017.06.015.

[23] C. Latimer, J. Kópházi, M. D. Eaton, R. G. McClarren, A geometry conforming isogeometric method for the self-adjoint angular flux (SAAF) form of the neutron transport equation with a discrete ordinate $\left(\mathrm{S}_{\mathrm{N}}\right)$ angular discretisation, Annals of Nuclear Energy 136 (2020) 107049. doi:10.1016/j.anucene.2019.107049.

[24] C. Latimer, J. Kópházi, M. D. Eaton, R. G. McClarren, A geometry conforming, isogeometric, weighted least squares (WLS) method for the neutron transport equation with discrete ordinate $\left(\mathrm{S}_{\mathrm{N}}\right)$ angular discretisation, Progress in Nuclear Energy 121 (2020) 103238. doi:10.1016/j.pnucene.2019.103238.

URL https://linkinghub.elsevier.com/retrieve/pii/S0149197019303439

[25] E. Wachspress, A Rational Finite Element Basis, Academic Press, New York, 1975.

[26] N. Sukumar, A. Tabarraei, Conforming polygonal finite elements, International Journal for Numerical Methods in Engineering 61 (12) (2004) 2045-2066. doi:10.1002/nme.1141.

[27] N. Sukumar, E. A. Malsch, Recent advances in the construction of polygonal finite element interpolants, Archives of Computational Methods in Engineering 13 (1) (2006) 129-163. doi:10.1007/bf02905933.

[28] A. Tabarraei, N. Sukumar, Extended finite element method on polygonal and quadtree meshes, Computer Methods in Applied Mechanics and Engineering 197 (5) (2008) 425438. doi:10.1016/j.cma.2007.08.013. 
[29] T. S. Bailey, M. L. Adams, B. Yang, M. R. Zika, A piecewise linear finite element discretization of the diffusion equation for arbitrary polyhedral grids, Journal of Computational Physics 227 (8) (2008) 3738-3757. doi:10.1016/j.jcp.2007.11.026.

[30] J. C. Ragusa, Discontinuous finite element solution of the radiation diffusion equation on arbitrary polygonal meshes and locally adapted quadrilateral grids, Journal of Computational Physics 280 (2015) 195-213. doi:10.1016/j.jcp.2014.09.013.

[31] L. B. da Veiga, K. Lipnikov, G. Manzini, Arbitrary-order nodal mimetic discretizations of elliptic problems on polygonal meshes, SIAM Journal on Numerical Analysis 49 (5) (2011) 1737-1760. doi:10.1137/100807764.

[32] L. B. da Veiga, G. Manzini, A higher-order formulation of the mimetic finite difference method, SIAM Journal on Scientific Computing 31 (1) (2008) 732-760. doi:10.1137/ 080717894.

[33] K. Lipnikov, G. Manzini, M. Shashkov, Mimetic finite difference method, Journal of Computational Physics 257 Part B (2014) 1163-1227. doi:10.1016/j.jcp.2013.07.031.

[34] Y. Kuznetsov, K. Lipnikov, M. Shashkov, The mimetic finite difference method on polygonal meshes for diffusion-type problems, Computational Geosciences 8 (4) (2004) 301-324. doi:10.1007/s10596-004-3771-1.

[35] J. Hyman, J. Morel, M. Shashkov, S. Steinberg, Mimetic finite difference methods for diffusion equations, Computational Geosciences 6 (3/4) (2002) 333-352. doi:10.1023/a: 1021282912658.

[36] L. B. da Veiga, G. Manzini, A virtual element method with arbitrary regularity, IMA Journal of Numerical Analysis 34 (2) (2013) 759-781. doi:10.1093/imanum/drt018.

[37] A. Russo, On the choice of the internal degrees of freedom for the nodal virtual element method in two dimensions, Computers \& Mathematics with Applications 72 (8) (2016) 1968-1976. doi:10.1016/j. camwa.2016.03.016.

[38] B. Ahmad, A. Alsaedi, F. Brezzi, L. D. Marini, A. Russo, Equivalent projectors for virtual element methods, Computers \& Mathematics with Applications 66 (3) (2013) 376-391. doi:10.1016/j.camwa.2013.05.015.

[39] L. B. da Veiga, F. Dassi, A. Russo, High-order virtual element method on polyhedral meshes, Computers \& Mathematics with Applications 74 (5) (2017) 1110-1122. doi: $10.1016 / j$. camwa.2017.03.021.

[40] A. Cangiani, G. Manzini, O. J. Sutton, Conforming and nonconforming virtual element methods for elliptic problems, IMA Journal of Numerical Analysis 37 (3) (2016) 1317-1354. doi:10.1093/imanum/drw036.

[41] B. A. de Dios, K. Lipnikov, G. Manzini, The nonconforming virtual element method, ESAIM: Mathematical Modelling and Numerical Analysis 50 (3) (2016) 879-904. doi: $10.1051 / \mathrm{m} 2 \mathrm{an} / 2015090$.

[42] X. Liu, J. Li, Z. Chen, A nonconforming virtual element method for the stokes problem on general meshes, Computer Methods in Applied Mechanics and Engineering 320 (2017) 694-711. doi:10.1016/j.cma.2017.03.027. 
[43] S. Berrone, A. Borio, G. Manzini, SUPG stabilization for the nonconforming virtual element method for advection-diffusion-reaction equations, Computer Methods in Applied Mechanics and Engineering 340 (2018) 500-529. doi:10.1016/j.cma.2018.05.027.

[44] M. F. Benedetto, S. Berrone, A. Borio, S. Pieraccini, S. Scialò, Order preserving SUPG stabilization for the virtual element formulation of advection-diffusion problems, Computer Methods in Applied Mechanics and Engineering 311 (2016) 18-40. doi:10.1016/j.cma. 2016.07 .043$.

[45] L. B. da Veiga, F. Brezzi, L. D. Marini, A. Russo, $H$ (div) and $H$ (curl)-conforming virtual element methods, Numerische Mathematik 133 (2) (2015) 303-332. doi:10.1007/ s00211-015-0746-1.

[46] F. Dassi, L. Mascotto, Exploring high-order three dimensional virtual elements: bases and stabilizations, Computers \& Mathematics with Applications 75 (9) (2018) 3379-3401. doi:10.1016/j.camwa.2018.02.005.

[47] L. Mascotto, Ill-conditioning in the virtual element method: stabilizations and bases, Numerical Methods for Partial Differential Equations 34 (4) (2018) 1258-1281. doi:10. 1002/num. 22257.

[48] S. Berrone, A. Borio, Orthogonal polynomials in badly shaped polygonal elements for the virtual element method, Finite Elements in Analysis and Design 129 (2017) 14-31. doi:10.1016/j.finel.2017.01.006.

[49] L. B. da Veiga, F. Brezzi, L. D. Marini, A. Russo, Virtual element method for general second-order elliptic problems on polygonal meshes, Mathematical Models and Methods in Applied Sciences 26 (04) (2016) 729-750. doi:10.1142/s0218202516500160.

[50] L. B. da Veiga, F. Brezzi, L. D. Marini, A. Russo, Mixed virtual element methods for general second order elliptic problems on polygonal meshes, ESAIM: Mathematical Modelling and Numerical Analysis 50 (3) (2016) 727-747. doi:10.1051/m2an/2015067.

[51] G. Vacca, Virtual element methods for hyperbolic problems on polygonal meshes, Computers \& Mathematics with Applications 74 (5) (2017) 882-898. doi:10.1016/j.camwa. 2016.04 .029$.

[52] G. Vacca, L. B. da Veiga, Virtual element methods for parabolic problems on polygonal meshes, Numerical Methods for Partial Differential Equations 31 (6) (2015) 2110-2134. doi:10.1002/num.21982.

[53] P. F. Antonietti, G. Manzini, M. Verani, The conforming virtual element method for polyharmonic problems, Computers and Mathematics with Applications, In PressarXiv: http://arxiv.org/abs/1811.04317v1.

[54] P. F. Antonietti, G. Manzini, M. Verani, The fully nonconforming virtual element method for biharmonic problems, Mathematical Models and Methods in Applied Sciences 28 (02) (2017) 387-407. doi:10.1142/s0218202518500100.

[55] A. L. Gain, C. Talischi, G. H. Paulino, On the virtual element method for three-dimensional linear elasticity problems on arbitrary polyhedral meshes, Computer Methods in Applied Mechanics and Engineering 282 (2014) 132-160. doi:10.1016/j.cma.2014.05.005. 
[56] F. Brezzi, L. D. Marini, Virtual element methods for plate bending problems, Computer Methods in Applied Mechanics and Engineering 253 (2013) 455-462. doi:10.1016/j. cma. 2012.09.012.

[57] F. Wang, H. Wei, Virtual element method for simplified friction problem, Applied Mathematics Letters 85 (2018) 125-131. doi:10.1016/j.aml.2018.06.002.

[58] P. Wriggers, W. T. Rust, B. D. Reddy, A virtual element method for contact, Computational Mechanics 58 (6) (2016) 1039-1050. doi:10.1007/s00466-016-1331-x.

[59] F. Aurenhammer, R. Klein, D.-T. Lee, Voronoi Diagrams and Delaunay Triangulations, WORLD SCIENTIFIC, 2013. doi:10.1142/8685.

URL https://www.worldscientific.com/worldscibooks/10.1142/8685

[60] A. Okabe, B. Boots, K. Sugihara, S. N. Chiu, D. G. Kendall (Eds.), Spatial Tessellations, Wiley Series in Probability and Statistics, John Wiley \& Sons, Inc., Hoboken, NJ, USA, 2000. doi:10.1002/9780470317013.

URL http://doi.wiley.com/10.1002/9780470317013

[61] S. Landier, Boolean operations on arbitrary polygonal and polyhedral meshes, ComputerAided Design 85 (2017) 138-153. doi:10.1016/j.cad.2016.07.013.

URL https://linkinghub.elsevier.com/retrieve/pii/S0010448516300847

[62] S. Y. Lee, Polyhedral Mesh Generation and A Treatise on Concave Geometrical Edges, Procedia Engineering 124 (2015) 174-186. doi:10.1016/j.proeng. 2015.10.131.

URL https://linkinghub.elsevier.com/retrieve/pii/S1877705815032324

[63] D. Contreras, N. Hitschfeld-Kahler, Generation of Polyhedral Delaunay Meshes, Procedia Engineering 82 (2014) 291-300. doi:10.1016/j.proeng.2014.10.391.

URL https://linkinghub.elsevier.com/retrieve/pii/S1877705814016701

[64] Y. Liu, A. A. Saputra, J. Wang, F. Tin-Loi, C. Song, Automatic polyhedral mesh generation and scaled boundary finite element analysis of STL models, Computer Methods in Applied Mechanics and Engineering 313 (2017) 106-132. doi:10.1016/j .cma.2016.09.038. URL https://linkinghub.elsevier.com/retrieve/pii/S0045782516306326

[65] S. Kim, D. Sohn, S. Im, Construction of polyhedral finite element meshes based upon marching cube algorithm, Advances in Engineering Software 128 (2019) 98-112. doi: $10.1016 / j$. advengsoft. 2018.11.014.

URL https://linkinghub.elsevier.com/retrieve/pii/S0965997818303053

[66] J. Ferguson, J. Kópházi, M. D. Eaton, Polygonal Virtual Element Spatial Discretisation Methods for the Neutron Diffusion Equation With Applications in Nuclear Reactor Physics, in: Volume 3: Nuclear Fuel and Material, Reactor Physics, and Transport Theory, American Society of Mechanical Engineers, 2018. doi:10.1115/ICONE26-81317. URL https://asmedigitalcollection.asme.org/ICONE/proceedings/ICONE26/ 51456/London, England/272707

[67] L. B. da Veiga, F. Brezzi, L. D. Marini, A. Russo, The hitchhikers guide to the virtual element method, Mathematical Models and Methods in Applied Sciences 24 (08) (2014) 1541-1573. doi:10.1142/s021820251440003x. 
[68] L. R. S. Susanne C. Brenner, The Mathematical Theory of Finite Element Methods, Springer-Verlag GmbH, 2007.

URL https://www.ebook.de/de/product/7043700/susanne_c_brenner_l_ridgway_ scott_the_mathematical_theory_of_finite_element_methods.html

[69] L. B. da Veiga, F. Brezzi, L. D. Marini, A. Russo, Virtual Element Implementation for General Elliptic Equations, in: Building Bridges: Connections and Challenges in Modern Approaches to Numerical Partial Differential Equations, Springer International Publishing, 2016, pp. 39-71. doi:10.1007/978-3-319-41640-3_2.

[70] E. H. James J. Duderstadt, Nuclear Reactor Analysis, John Wiley \& Sons, Inc., 1976.

URL https://www.ebook.de/de/product/3597336/james_j_duderstadt_e_ hamilton_nuclear_reactor_analysis.html

[71] E. Wachspress, Iterative Solution of Elliptic Systems: and Applications to the Neutron Diffsusion Equations of Reactor Physics, Prentice-Hall, 1966.

[72] A. R. Owens, J. Kópházi, J. A. Welch, M. D. Eaton, Energy dependent mesh adaptivity of discontinuous isogeometric discrete ordinate methods with dual weighted residual error estimators, Journal of Computational Physics 335 (2017) 352-386. doi:10.1016/j.jcp. 2017.01 .035 .

[73] S. Balay, S. Anhyankar, M. F. Adams, J. Brown, P. Brune, D. Buschleman, D. Kaushik, M. G. Knepley, D. A. May, L. C. McInnes, R. T. Mills, T. Munson, K. Rupp, P. Sanan, B. F. Smith, S. Zampini, H. Zhang, PETSc Users Manual, Tech. rep., Argonne National Laborotary (2020).

URL https://www.mcs.anl.gov/petsc

[74] Y. Saad, Iterative Methods for Sparse Linear Systems, 2nd Edition, Society for Industrial and Applied Mathematics, 2003. doi:10.1137/1.9780898718003.

URL http://epubs.siam.org/doi/book/10.1137/1.9780898718003

[75] Q. Du, V. Faber, M. Gunzburger, Centroidal Voronoi Tessellations: Applications and Algorithms, SIAM Review 41 (4) (1999) 637-676. doi:10.1137/S0036144599352836.

URL http://epubs.siam.org/doi/10.1137/S0036144599352836

[76] C. Talischi, G. H. Paulino, A. Pereira, I. F. M. Menezes, PolyMesher: a general-purpose mesh generator for polygonal elements written in MATLAB, Structural and Multidisciplinary Optimization 45 (3) (2012) 309-328. doi:10.1007/s00158-011-0706-z.

[77] Argonne Code Center, Benchmark problem book ANL-7416 supplement 2, mathematics and computers (UC-32), Tech. rep., Argonne National Laboratory (1977).

[78] E. E. Lewis, M. A. Smith, N. Tsoulfanidis, G. Palmiotti, T. A. Taiwo, R. N. Blomquist, Benchmark on Deterministic Transport Calculations Without Spatial Homogenisation: A 2-D/3-D MOX Fuel Assembly Benchmark, Tech. Rep. NEA/NSC/ DOC(2003)16, Nuclear Energy Agency (NEA), Organisation for Economic Co-operation and Development (2003).

[79] L. B. da Veiga, A. Russo, G. Vacca, The virtual element method with curved edges, ESAIM: Mathematical Modelling and Numerical Analysis 53 (2) (2019) 375-404. doi: 10.1051/m2an/2018052. 
[80] R. Garimella, M. Kucharik, M. Shashkov, An efficient linearity and bound preserving conservative interpolation (remapping) on polyhedral meshes, Computers \& Fluids 36 (2) (2007) 224-237. doi:10.1016/j.compfluid.2006.01.014.

URL https://linkinghub.elsevier.com/retrieve/pii/S0045793006000351

[81] S. Menon, D. P. Schmidt, Conservative interpolation on unstructured polyhedral meshes: An extension of the supermesh approach to cell-centered finite-volume variables, Computer Methods in Applied Mechanics and Engineering 200 (41-44) (2011) 2797-2804. doi:10. 1016/j.cma.2011.04.025.

URL https://linkinghub.elsevier.com/retrieve/pii/S0045782511001666

[82] J. D. Franklin, J. S. Lee, A high quality interpolation method for colocated polyhedral/polygonal control volume methods, Computers \& Fluids 39 (6) (2010) 1012-1021. doi:10.1016/j.compfluid.2010.01.010.

URL https://linkinghub.elsevier.com/retrieve/pii/S0045793010000113

[83] J.-L. G. Alexandre Ern, Theory and Practice of Finite Elements, Springer New York, 2010.

URL https://www.ebook.de/de/product/13413656/alexandre_ern_jean_luc_ guermond_theory_and_practice_of_finite_elements.html

[84] C. Pozrikidis, Introduction to Finite and Spectral Element Methods Using MATLAB, Second Edition, CRC Press, Taylor and Francis Group, Boca Raton, Florida, USA, 2014. URL https://www .crcpress.com/Introduction-to-Finite-and-Spectral-Element-Methods Pozrikidis/p/book/9781482209150 